\title{
Promotion of $\beta$-Catenin/Forkhead Box Protein 0 Signaling Mediates Epithelial Repair in Kidney Injury
}

Padmashree Rao, ${ }^{*}$ Xi Qiao, ${ }^{\star \dagger}$ Winston Hua, ${ }^{*}$ Min Hu, ${ }^{*}$ Mariah Tahan, ${ }^{*}$ Titi Chen, ${ }^{*}$ Hong Yu, ${ }^{*}$ Xiaojun Ren, ${ }^{* \dagger}$ Qi Cao, ${ }^{*}$ Yiping Wang, ${ }^{*}$ Ying Yang, ${ }^{* \dagger}$ Yuan M. Wang, ${ }^{\ddagger}$ Vincent W. Lee, ${ }^{\star \S}$ Stephen I. Alexander, ${ }^{\ddagger}$ David C. Harris, ${ }^{* \S}$ and Guoping Zheng ${ }^{\star}$

\begin{abstract}
From the Centre for Transplant and Renal Research, * Westmead Institute for Medical Research, The University of Sydney, Westmead, New South Wales, Australia; the Department of Nephrology, Shanxi Medical University, ${ }^{\dagger}$ Taiyuan, China; the Centre for Kidney Research, Children's Hospital at Westmead, Australia; and the Department of Nephrology, Westmead Hospital, ${ }^{\S}$ Australia
\end{abstract} Accepted for publication
March 5, 2021.
Address correspondence to
Guoping Zheng, Ph.D., Centre
for Transplantation and Renal
Research, The Westmead Insti-
tute for Medical Research, The
University of Sydney, 176
Hawkesbury Rd., Westmead,
NSW 2145, Australia. E-mail:
guoping.zheng@sydney.edu.au.

\begin{abstract}
Fibrosis is characterized by progressively excessive deposition of matrix components and may lead to organ failure. Transforming growth factor- $\beta$ (TGF- $\beta$ ) is a key cytokine involved in tissue repair and fibrosis. TGF- $\beta$ 's profibrotic signaling pathways converge at activation of $\beta$-catenin. $\beta$-Catenin is an important transcription cofactor whose function depends on its binding partner. Promoting $\beta$-catenin binding to forkhead box protein 0 (Foxo) via inhibition of its binding to T-cell factor (TCF) reduces kidney fibrosis in experimental murine models. Herein, we investigated whether $\beta$-catenin/Foxo diverts TGF- $\beta$ signaling from profibrotic to physiological epithelial healing. In an in vitro model of wound healing (scratch assay), and in an in vivo model of kidney injury, unilateral renal ischemia reperfusion, TGF- $\beta$ treatment in combination with either ICG-001 or iCRT3 ( $\beta$-catenin/TCF inhibitors) increased $\beta$ catenin/Foxo interaction, increased scratch closure by increased cell proliferation and migration, reduced the TGF- $\beta$-induced mesenchymal differentiation, and healed the ischemia reperfusion injury with less fibrosis. In addition, administration of ICG-001 or iCRT3 reduced the contractile activity induced by TGF- $\beta$ in $\mathrm{C} 1.1$ cells. Together, our results indicate that redirection of $\beta$-catenin binding from TCF to Foxo promotes $\beta$-catenin/Foxo-mediated epithelial repair. Targeting $\beta$-catenin/Foxo may rebuild normal structure of injured kidney. (Am J Pathol 2021, 191: 993-1009; https://doi.org/ 10.1016/j.ajpath.2021.03.005)
\end{abstract}

Fibrosis (scarring) is a pathologic wound repair process characterized by excessive deposition of extracellular matrix (ECM) components secreted by increasing numbers of myofibroblasts, with destruction of normal mesenchymal structure. ${ }^{1}$ This is in contrast to physiological repair, where the wound heals with restoration of normal tissue structure without fibrosis. ${ }^{2}$ Fibrosis impedes tissue repair, regeneration, complete restoration of original tissue architecture, and reconstitution of organ function. ${ }^{3}$ The balance between tissue repair and fibrosis following kidney injury depends on the severity and duration of injury as well as the responses of kidney tubules to injury. ${ }^{4}$ Renal tubular epithelial cells are extremely susceptible to kidney injury. ${ }^{5}$ In response to inflammation and injury, activated myofibroblasts express high levels of $\alpha$-smooth muscle actin ( $\alpha$-SMA) and produce large amounts of ECM components, presumably to heal the injury. ${ }^{6,7}$ Transforming growth factor- $\beta$ (TGF- $\beta$ ) promotes wound repair but is also the key cytokine causing organ fibrosis. Targeting the signaling pathways of TGF- $\beta$ that promote kidney tubule repair rather than fibrosis could be an important strategy for separating these opposing effects. ${ }^{3}$

$\beta$-Catenin is a central mediator in TGF- $\beta^{\prime} \mathrm{s}$ profibrotic signaling pathways. ${ }^{8}$ Stabilized $\beta$-catenin translocates to the nucleus and binds to T-cell factor (TCF). ${ }^{9} \quad \beta$-Catenin/ $\mathrm{TCF}-$ mediated signaling promotes the migration,

\footnotetext{
Supported by the National Health and Medical Research Council of Australia project grants APP1046647 and 1141235; and an Australian Government Research Training Program Scholarship (P.R.).

Disclosures: None declared.
} 
proliferation, and prolonged or excessive activation of mesenchymal cells, leading to fibrosis and organ dysfunction. ${ }^{10,11}$ On the other hand, $\beta$-catenin also binds to forkhead box protein $\mathrm{O}$ (Foxo). Foxo plays a role in cellular processes, such as $G_{1}$ cell cycle arrest/DNA repair, and has antioxidant properties, which may be protective in wound repair and fibrosis. ${ }^{4,12,13}$ Ponugoti et al ${ }^{12}$ showed that Foxo1 is required to orchestrate cellular events promoting a wound healing phenotype and prevention of oxidative stress. Hence, we hypothesize that targeted inhibition of $\beta$-catenin/ TCF binding increases $\beta$-catenin/Foxo interaction and consequent wound repair action of TGF- $\beta$.

In the current study, the role of $\beta$-catenin/Foxo in wound healing was investigated using Clustered regularly interspaced palindromic repeats (CRISPR/Cas9)-mediated deletion of the Foxo1 gene in tubular epithelial $\mathrm{C} 1.1$ cells and in the kidney healing model of unilateral ischemia reperfusion (UIR). The results indicate that $\beta$-catenin/Foxo promotes the wound healing effect of TGF- $\beta$ in a physiological epithelial repair manner.

\section{Materials and Methods}

\section{Cell Cultures}

A murine proximal tubule-like epithelial cell line termed C1.1 was a kind gift of Drs. Rudolph Wuthrich and Patricia Wahl (University of Zurich, Zurich, Switzerland). ${ }^{14}$ C1.1 cells were cultured in K1 medium [Dulbecco's modified Eagle's medium:Ham's F12 medium (1:1 v/v) (Invitrogen, Carlsbad, CA), $1 \mathrm{~mol} / \mathrm{L}$ HEPES (Invitrogen), Non-Essential Amino Acid (NEAA) medium (Invitrogen), $5 \mathrm{mg} / \mathrm{mL}$ insulin (Sigma, St. Louis, MO), $1.25 \mathrm{ng} / \mathrm{mL}$ prostaglandin E1 (Cayman Chemicals, Ann Arbor, MI), 0.0338 ng/mL 3,3,5triiodothyronine (Sigma), $5 \mathrm{mg} / \mathrm{mL}$ hydrocortisone (Sigma), $1.73 \mathrm{ng} / \mathrm{mL}$ transferrin (Sigma), $18 \mathrm{ng} / \mathrm{mL}$ sodium selenite (Sigma), and 5\% fetal bovine serum (Sigma-Aldrich, St. Louis, MO)] at $37^{\circ} \mathrm{C}$, in $5 \% \mathrm{CO}_{2}$. For treatment of cells, serum-supplemented media were replaced with serum-free media.

\section{Generation of Foxo1 K0 Cell Line and TCF1 K0 Cell Line by CRISPR/Cas9}

Foxol knockout (KO) $\mathrm{C} 1.1$ cell line was produced by cotransfection of $\mathrm{C} 1.1$ cells with the Foxol CRISPR/Cas9 KO plasmid and a Foxo1 Homology directed repair (HDR) plasmid (Santa Cruz Biotechnology, Inc., Dallas, TX), following the manufacturer's instructions. In brief, C1.1 cells were transfected with the plasmids using UltraCruz transfection reagent (Santa Cruz Biotechnology, Inc.) and incubated under normal conditions for 24 hours. Media were replaced 24 hours after transfection. After two passages of the cells, Foxol KO C1.1 cells were selected with media containing puromycin antibiotic (2 $\mu \mathrm{g} / \mathrm{mL})$.
Similarly, TCF 1 gene was knocked out using TCF-1 Double Nickase Plasmid (Santa Cruz Biotechnology, Inc.).

KO was verified by Western blot analysis of Foxo1 and TCF1 expression and fluorescence microscopy for the Red Fluorescent Protein (RFP)/Green Fluorescent Protein (GFP) signal. ${ }^{15}$

\section{In Vitro Scratch Assay}

Control C1.1, Foxo1 KO C1.1, and TCF1 KO C1.1 cells were grown to confluence on (Thermo Scientific, Waltham, MA USA). Confluent cells were scratched using sterile $200 \mu \mathrm{L}$ pipette tips across each well, generating a cell-free area, based on the technique described by Liang et al. ${ }^{16}$ Cells were washed with Dulbecco's phosphatebuffered saline (PBS) to remove loose cells. Foxol KO $\mathrm{C} 1.1$ and TCF1 KO C1.1 cells were transiently transfected with F-TrCP-Ecad plasmid by Lipofectamine 2000 (Invitrogen), according to the manufacturer's protocol. F-TrCP-Ecad plasmid construct encodes a chimeric protein with the binding domain of E-cadherin fused to the $\beta$-transducin repeat-containing protein $(\beta \operatorname{TrCP})$ ubiquitin-protein ligase. The cells were treated with recombinant human TGF- $\beta 1$ (rhTGF- $\beta 1 ; 3 \mathrm{ng} / \mathrm{mL}$; Biosource, Camarillo, CA) either with or without inhibitors, ICG-001 (5 $\mu \mathrm{mol} / \mathrm{L}$; Sigma) or iCRT3 (10 $\mu \mathrm{mol} / \mathrm{L}$; Sigma), in K1 medium supplemented with $1 \%$ bovine serum albumin for 48 hours. After 48 hours of treatment, cells were washed twice with Dulbecco's PBS (Thermo Scientific, Waltham, MA USA) and processed for Sirius red collagen detection assay, proximity ligation assay (PLA), or fluorescence.

\section{Image Capture and Data Analysis for Scratch Assay}

Immediately after the scratch ( 0 hours) and at 12 -hour intervals, images of the scratched area were captured using phase contrast microscopy. Alternatively, live cell imaging was performed using a Carl Zeiss microscope, Oberkochen, Germany.

The area of scratch was quantified using an ImageJ version 1.52v software (NIH, Bethesda, MD; http://imagej. nih.gov/ij, last accessed December 16, 2020). The migration of cells toward the scratch was expressed as a percentage of wound closure.

$\%$ of wound closure $=\left[\left(\mathrm{A}_{\mathrm{t}}=0 \mathrm{hr}-\mathrm{A}_{\mathrm{t}}=\Delta \mathrm{hr}\right) / \mathrm{A}_{\mathrm{t}}=\right.$ o hours] X $100 .{ }^{17}$

where, $A_{t}=0$ hours is the area of wound at 0 hours, immediately after scratching, and $A_{t}=\Delta h r$ is the area of wound at $12,24,36$, or 48 hours after scratching.

\section{Sirius Red Collagen Detection Assay}

C1.1, Foxol KO C1.1, and TCF1 KO C1.1 cells with or without treatment and washed with PBS were used for collagen assay using Chondrex Sirius red collagen detection kit (Chondrex, Inc. Woodinville, WA) following the manufacturer's instructions. Briefly, $0.05 \mathrm{~mol} / \mathrm{L}$ acetic acid 
A

Scratch assay
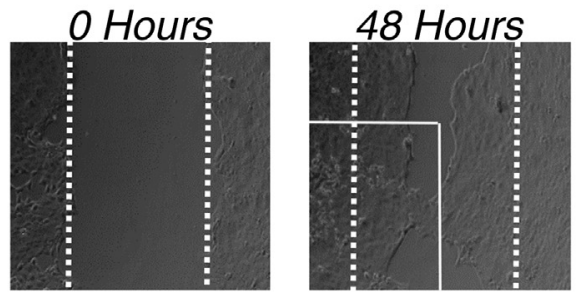

\section{B 48 Hours}
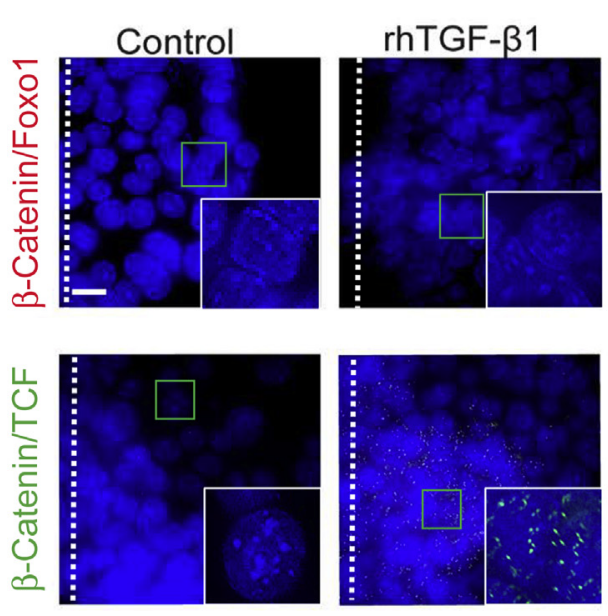

C

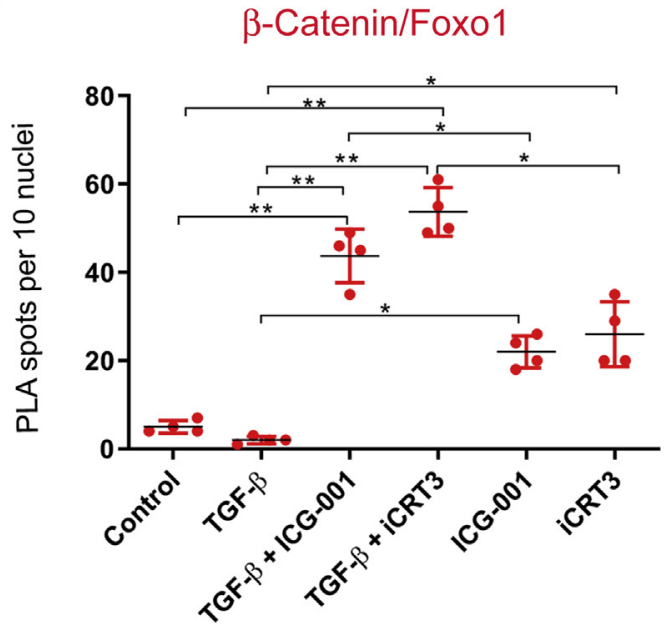

rhTGF- $\beta 1+$ ICG-001
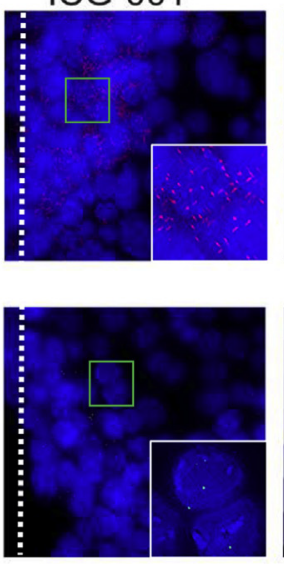

D
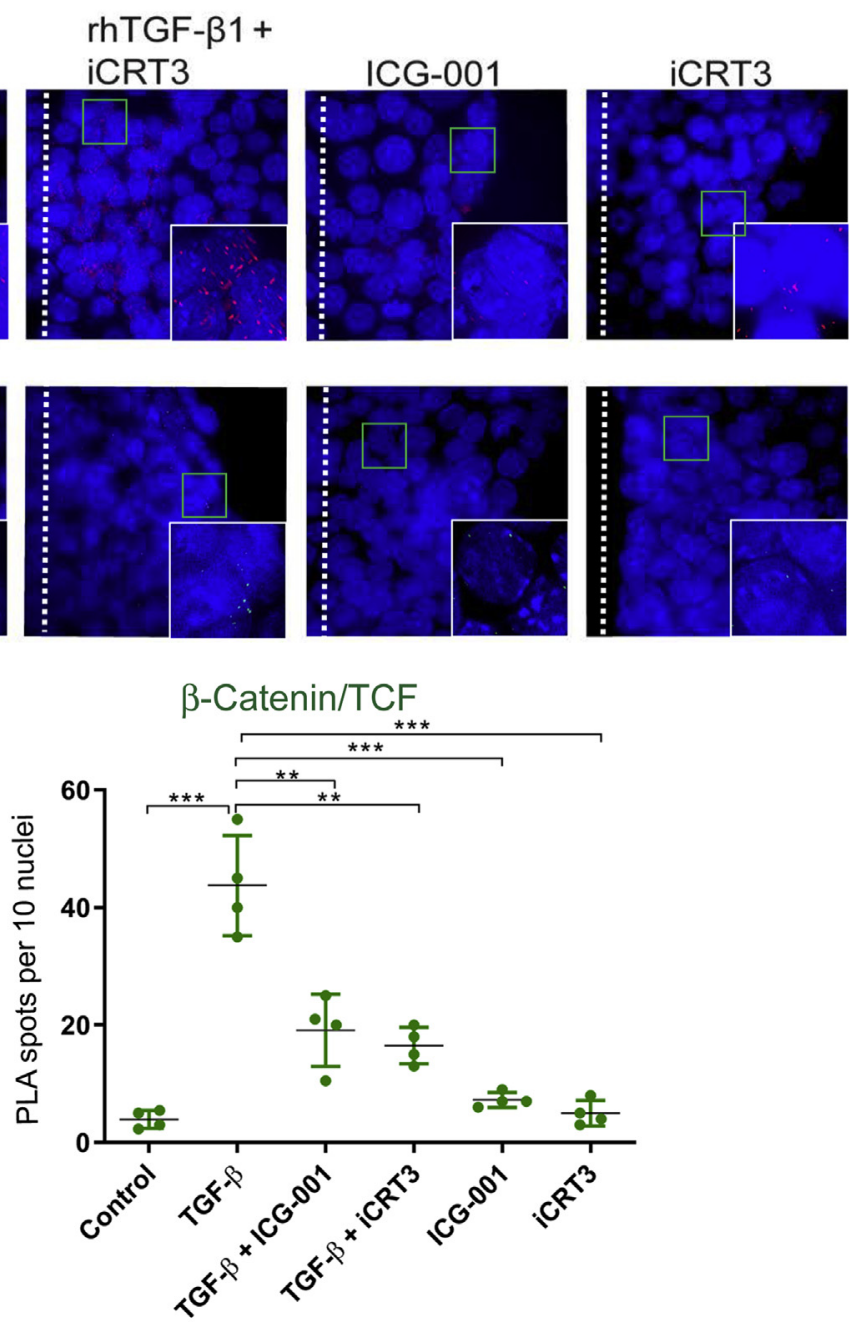

Figure 1 Proximity ligation assay (PLA) analysis of $\beta$-catenin/forkhead box protein 01 (Foxo1) and $\beta$-catenin/T-cell factor (TCF) interactions in scratch assay using C1.1 cells. A: Representative phase contrast images of WT C1.1 cells using in vitro scratch assay at 0 and 48 hours. The area between broken lines is the scratch wound. The solid lines outline the field are used in immunofluorescence imaging. B: Representative $\beta$-catenin/Foxo1 (red spot) and $\beta$-catenin/ TCF (green spot) interaction in control, recombinant human transforming growth factor- $\beta 1$ (rhTGF- $\beta 1$ ), rhTGF- $\beta 1$ and ICG-001, rhTGF- $\beta 1$ and iCRT3, ICG-001 alone, or iCRT3 alone treated C1.1 cells using scratch assay (cells to the right of broken lines are cells migrating after the scratch). Insets: Higher-resolution images of the smaller boxed areas outlined with solid green lines. Nuclei were stained with DAPI. C and D: Quantitation of PLA signals, using one-way analysis of variance, followed by Tukey post hoc test. Results are shown as means \pm SEM (C and D). $n=4$ (C and D). ${ }^{\star} P<0.05,{ }^{* *} P<0.01$, $* * * P<0.001$. Scale bar $=40 \mu \mathrm{m}(\mathbf{B})$. Original magnification, $\times 40(\mathbf{B})$.

was added, and the cell layer was scaped with cell scraper. A total of $1 \mathrm{mg} / \mathrm{mL}$ pepsin solution was added. A purified total collagen solution $(0.1 \mathrm{mg} / \mathrm{mL})$ was used as a standard. The insoluble complex of collagen and Sirius red dye was collected, solubilized, and then transferred to a 96well plate. OD values at $562 \mathrm{~nm}$ were measured. The collagen concentration was calculated using regression analysis. 


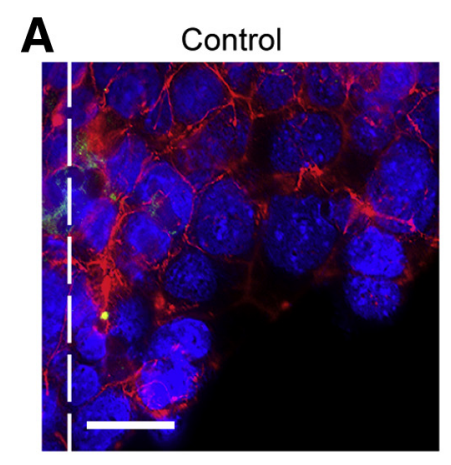

rhTGF- $\beta 1+$ EGCG

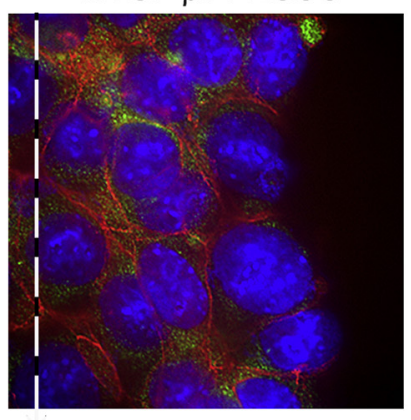

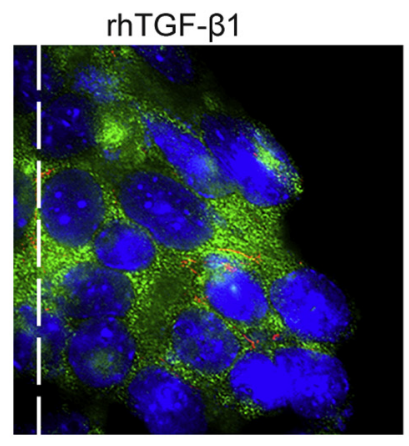

EGCG

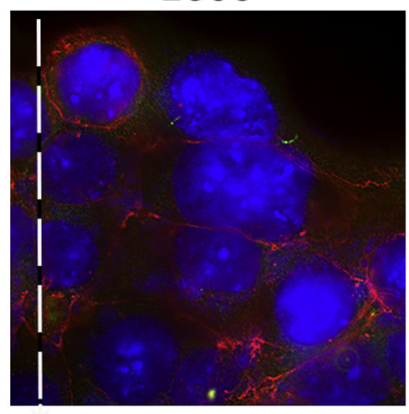

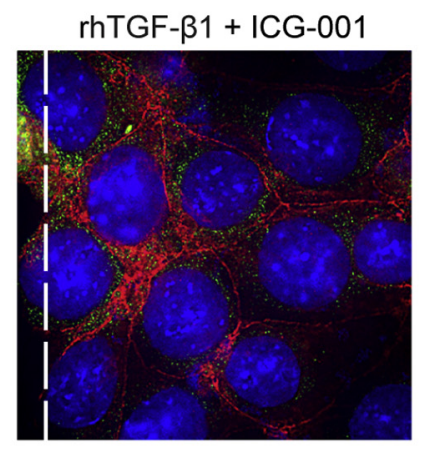

ICG-001

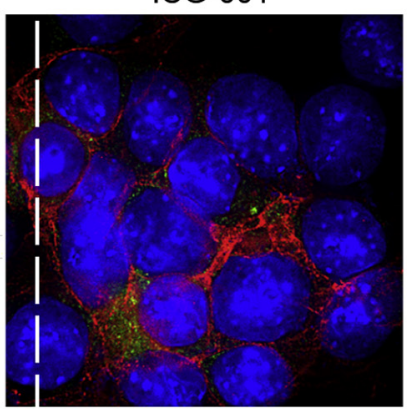

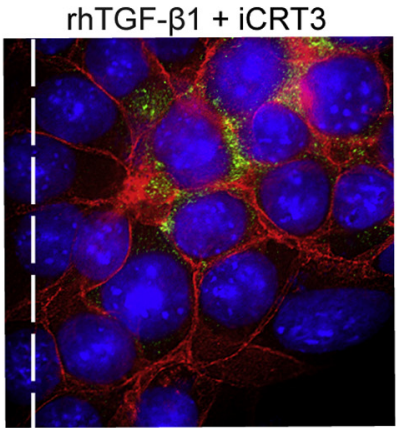

iCRT3

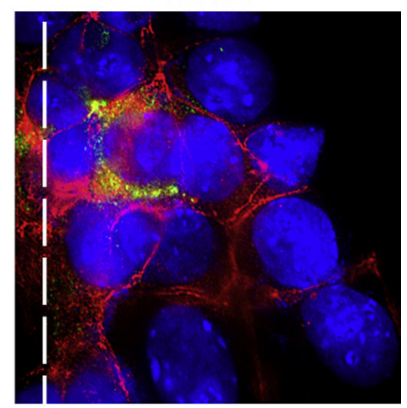

B

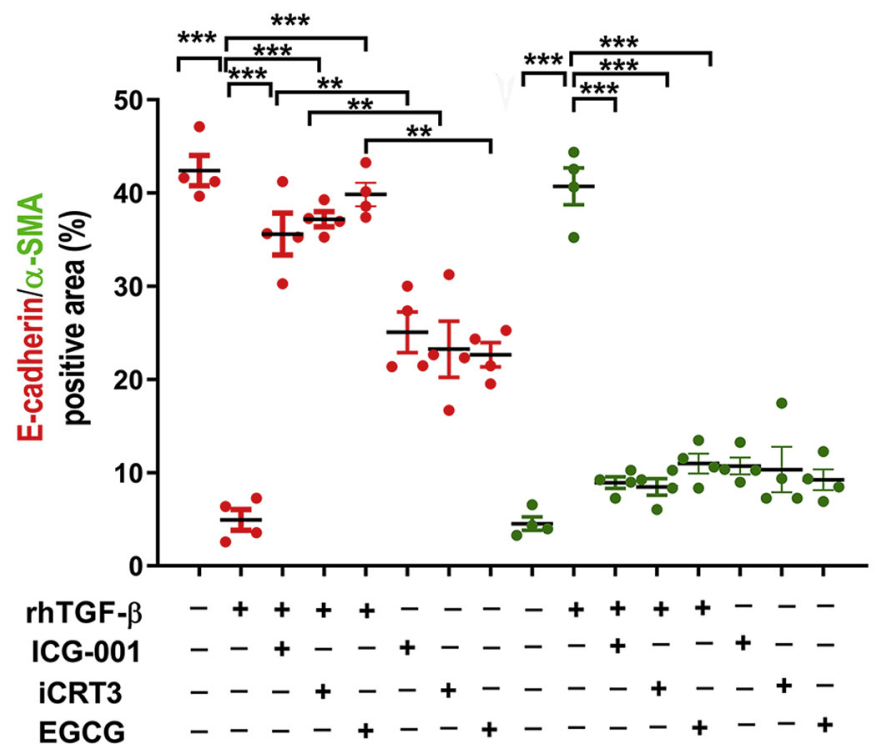

Figure 2 E-cadherin and $\alpha$-smooth muscle actin ( $\alpha$-SMA) expression in a scratch assay using C1.1 cells. A: Representative immunofluorescence images of E-cadherin (red) and $\alpha$-SMA (green) in the scratch assay (cells to the right of broken lines are cells migrating after the scratch) using C1.1 untreated cells (control) or cells treated with recombinant human transforming growth factor- $\beta 1$ (rhTGF- $\beta 1$ ), rhTGF- $\beta 1$ and ICG-001, rhTGF- $\beta 1$ and iCRT3, rhTGF- $\beta 1$ and epigallocatechin 3-gallate (EGCG), or treated with ICG-001, iCRT3, and EGCG alone. Nuclei were stained with DAPI. B: Quantitation of positive E-cadherin (red) and $\alpha$-SMA (green) staining area relative to the total area from 10 random microscopic fields was assessed with various treatments and compared by one-way analysis of variance, followed by Tukey post hoc test. Results are shown as means \pm SEM (B). $n=4$ (B). ${ }^{* *} P<0.01$, and ${ }^{* * *} P<0.001$. Scale bar $=40 \mu \mathrm{m}$ (A). Original magnification, $\times 60($ A).

\section{Collagen Gel Contraction Assay}

Collagen gel contraction assay was performed on control C1.1, Foxol KO C1.1, and TCF1 KO C1.1 cells using CytoSelect 24-well cell contraction assay kit (Cell Biolabs, San Diego, CA) following the manufacturer's instructions. First, collagen gel working solution was prepared and kept on ice. Control C1.1, Foxol KO C1.1, and TCF1 KO C1.1 cells were harvested and resuspended in K1 medium. Cell contraction matrix was prepared by mixing two parts of cell suspension and eight parts of cold collagen gel working solution. A total of $0.5 \mathrm{~mL}$ of cell contraction matrix solution was added to each well of the 24-well cell contraction plate, and incubated at $37^{\circ} \mathrm{C}$ for 1 hour. After collagen 
polymerization, $1 \mathrm{~mL}$ of $\mathrm{K} 1$ medium was added (with/ without treatment) to each well. Negative controls were wells that contain no cells in the matrix. Medium was changed daily and replaced with $0.5 \mathrm{~mL}$ fresh $\mathrm{K} 1$ medium. After 48 hours (the end point), the collagen gel size was quantified with a ruler. Results are expressed as a change in gel surface area. The contraction of the gel is expressed in percentages, with the surface area of the noncontracted gel serving as $100 \%$.

\section{Proximity Ligation Assay (Duolink)}

PLA experiments were performed on C1.1 cells (grown in a Nunc Lab-Tek II chamber slide system; ThermoFisher Scientific, Waltham, MA USA) and paraffin-embedded mouse kidney sections using the DUO ink kit (SigmaAldrich) following the manufacturer's instructions. Briefly, either cells or deparaffinized tissues were fixed with $4 \%$ paraformaldehyde (Sigma) in PBS for 10 minutes, permeabilized with $0.5 \%$ Triton X-100 (Sigma) in PBS, and incubated with blocking solution in a humidity chamber for 1 hour. The primary antibodies [anti-mouse $\beta$-catenin (BD Transduction Laboratories, Franklin Lakes, NJ) and antirabbit Foxo1 (Cell Signaling, Danvers, MA) or anti-mouse $\beta$-catenin and anti-rabbit TCF1 (Cell Signaling)] were incubated overnight. Negative controls were obtained by omitting one of the primary antibodies. Secondary antibodies conjugated with PLA probes were added and incubated. Ligation and amplification were performed. Each slide was mounted with Duolink In Situ Mounting Medium (Sigma-Aldrich) with DAPI and analyzed in a DeltaVision microscope (Olympus Corperation, Shinjuku-ku, Tokyo, Japan). PLA spots were counted in intervals of 10 or 50 nuclei in each of multiple (five to eight) microscopic fields per slide, and then used averaged numbers for the calculation of number of PLA spots per 10 or 50 nuclei.

\section{Mice}

Male C57BL/6 mice were purchased from the Animal Resource Center (Perth, WA, Australia). Male mice, aged 8 to 10 weeks, were used in all experiments. All mice were housed in the Biological Services Facility at The Westmead Institute for Medical Research (Westmead, NSW, Australia) under standard conditions in accordance with a protocol approved by the Animal Ethics Committee of Western Sydney Local Health District.

\section{Mouse Kidney UIR Injury Model}

Male mice were anesthetized by isoflurane and placed on a heating pad to keep body temperature constant of around $37^{\circ} \mathrm{C}$. Mice were then randomized to sham or UIR injury operation. Renal ischemia was induced by placing clamps over the left renal pedicle and was visually verified by the change in kidney color. After 45 minutes, the clamps were removed. The incision was closed in two layers, and the reperfusion was allowed to occur. Sham-treated mice were subjected to the same procedure but without renal pedicle clamping. The UIR injury model is a well-characterized model of tubular injury and repair. ${ }^{18,19}$ UIR and control mice were treated with rhTGF- $\beta 1(50 \mu \mathrm{g} / \mathrm{kg}$ intraperitoneally) or vehicle second daily, with or without ICG-001 (5 $\mathrm{mg} / \mathrm{kg}$ intraperitoneally) or iCRT3 $(10 \mathrm{mg} / \mathrm{kg}$ intraperitoneally) daily. For delayed treatment, rhTGF- $\beta 1$ with either ICG-001 or iCRT3 was administered starting 7 days after UIR. Dose and duration were selected according to previously published studies. ${ }^{20-23}$ After 21 days, the animals were sacrificed, and kidneys were collected for the assessment of kidney fibrosis.

\section{Immunohistochemical and Immunofluorescence Staining}

Paraffin-embedded kidney sections were used for Gomori trichrome staining according to the protocol provided by the manufacturer (Sigma-Aldrich) to assess the degree of renal fibrosis. The stained slides were scanned by Nanozoomer (Hamamatsu, Hamamatsu City, Shizuoka, Japan) and quantitated in a blinded manner. For immunofluorescence staining, paraffin-embedded kidney slides were used. Initially, slides were dewaxed, rehydrated, and fixed with methanol and acetone at 1:1 volume. Nonspecific binding was blocked with $2 \%$ bovine serum albumin (Sigma) in PBS. Kidney sections were incubated with anti- $\alpha$-SMA and E-cadherin (Cell Signaling) for double staining. To visualize the primary antibodies, sections were stained with goat anti-rabbit Alexa Fluor 568 (ThermoFisher Scientific) and/or goat anti-mouse Alexa Fluor 488 (Invitrogen) and counterstained with DAPI (Sigma). The fluorescent images were viewed under a DeltaVision Elite Microscope (Olympus Corperation, Shinjuku-ku, Tokyo, Japan) in a blinded manner. For quantitation, the positive staining area was calculated relative to the total area of image. The mean value of 10 randomly selected microscopic fields was calculated and graphed.

\section{Fluorescence Microscopy}

Both cell and animal tissue images were acquired using a DeltaVision Elite Microscope (Olympus X-71) (Olympus Corperation, Shinjuku-ku, Tokyo, Japan). All images were captured at room temperature using a CoolsSnapHQ2 (Roper Scientific, Trenton, New Jersey, United States) camera. Deconvolution (conserved ratio method) and other image processing were performed using SoftWoRx Suite 2.0 (Applied Precision, GE, Boston, Massachusetts, USA). ImageJ software was used to quantify the images. Figures were made using Illustrator software 2020 (Adobe Systems Inc., Mountain View, CA). 


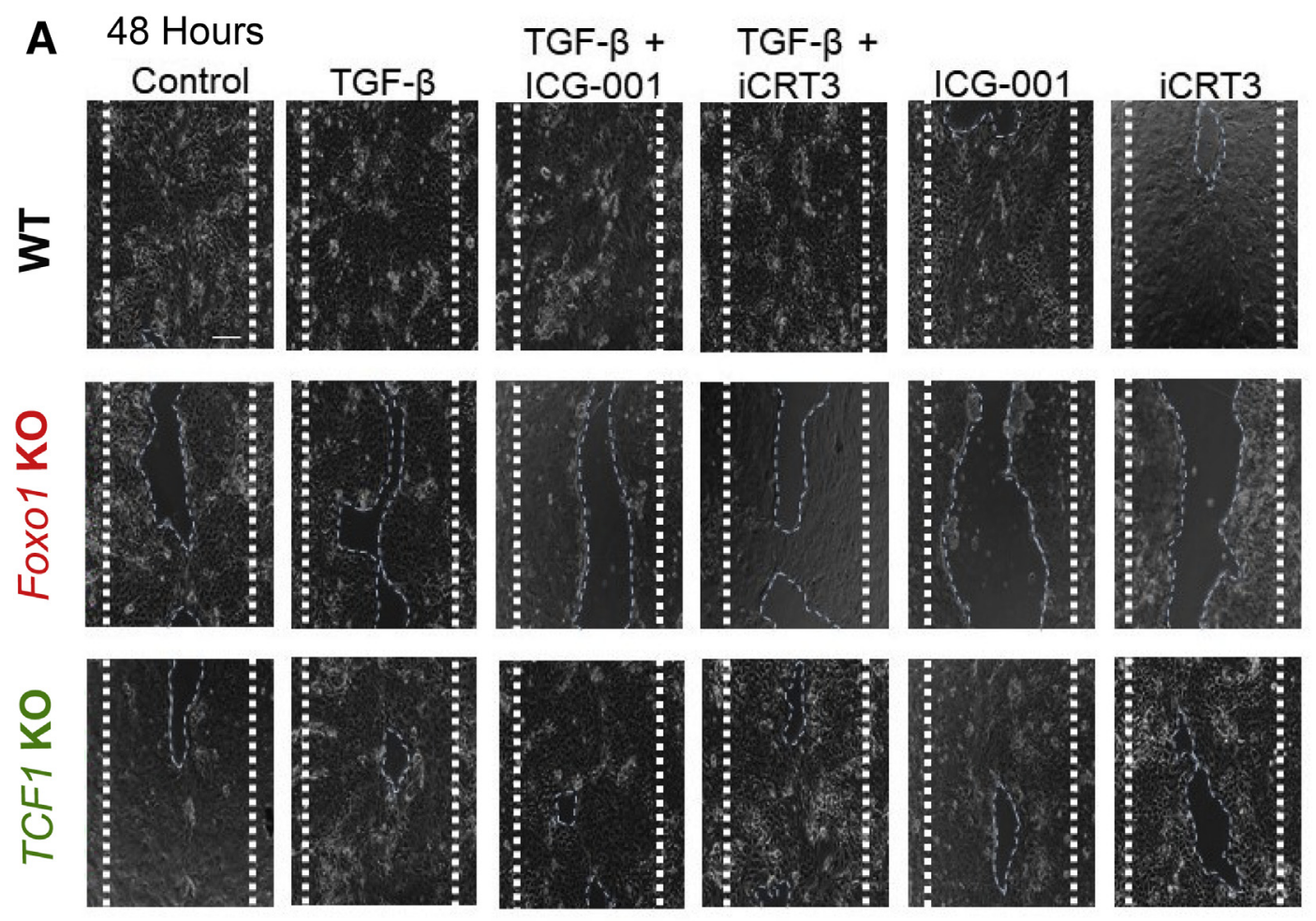

B

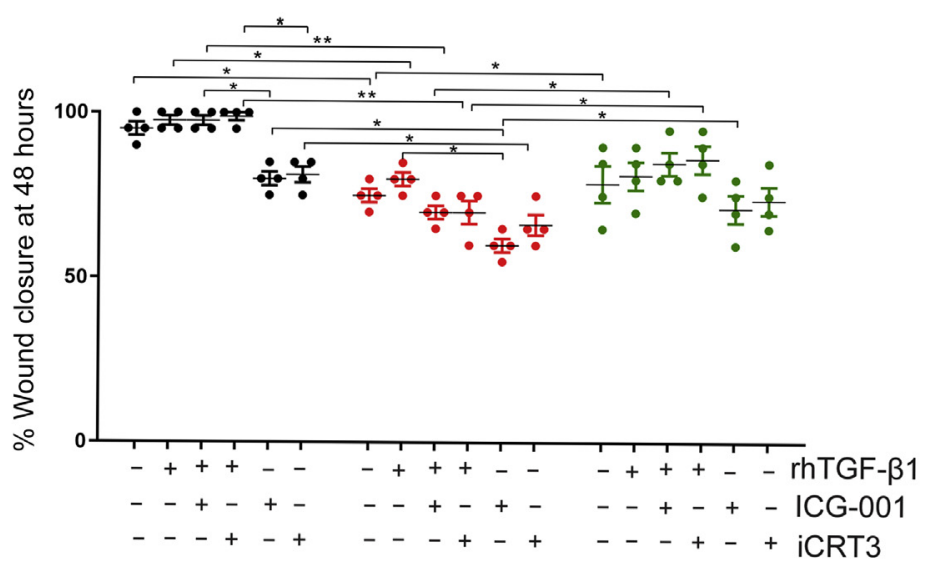

C

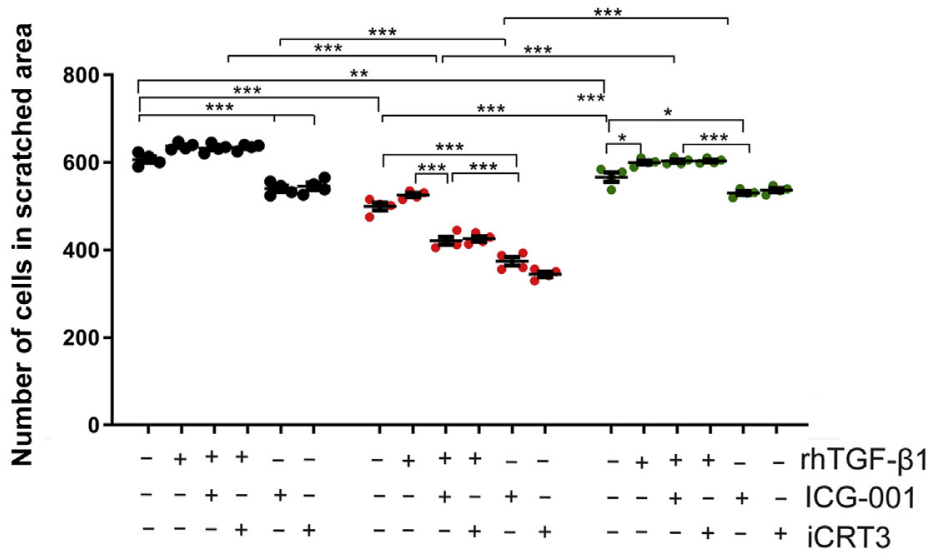




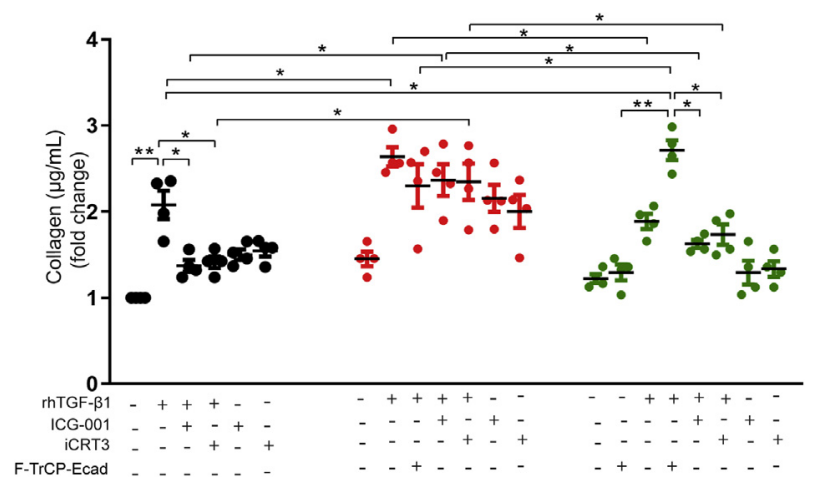

Figure 4 Sirius red collagen detection assay in wild-type (WT), Foxo1 knockout (KO), and TCF1 KO C1.1 cells. Quantitation of collagen (fold change) at 48 hours in WT (black dots), Foxo1 KO (red dots), and TCF1 KO C1.1 (green dots) with various treatments. F-TrCP-Ecad ( $\beta \operatorname{TrCP}$-E-cadherin chimera construct) were used for targeted degradation of cytosolic $\beta$-catein. Results are representative of four independent experiments. Statistical significance was determined by one-way analysis of variance, followed by Tukey post hoc test. ${ }^{*} P<0.05,{ }^{* *} P<0.01$. rhTGF- $\beta 1$, recombinant human transforming growth factor- $\beta 1$.

\section{Cytokine Cytometric Bead Array}

Tumor necrosis factor- $\alpha$, IL-6, IL-10, and IL-4 levels from kidney lysates were analyzed using BD cytometric bead array mouse cytokine kit following the manufacturer's instructions (BD Bioscience, Franklin Lakes, New Jersey, USA). Data were analyzed by BD FCAP Array (BD Bioscience).

\section{Statistical Analysis}

Statistical analyses of the data were performed using Graph Pad Prism 7 software. Comparison between groups was made using one-way analysis of variance, followed by Tukey post hoc test using Graph Pad Prism 7 software (GraphPad Software, San Diego, CA, USA). All data are expressed as means \pm SEM. $P$ $<0.05$ was considered statistically significant.

\section{Results}

Inhibition of TGF- $\beta$-Induced $\beta$-Catenin/TCF

Interactions Promotes $\beta$-Catenin Interaction with Foxo in C1.1 Cells

To assess the role of $\beta$-catenin/TCF or $\beta$-catenin/Foxo in cell migration, an in vitro scratch assay was performed using C1.1 cells, a murine proximal tubule-like epithelial cell line. First, a PLA as performed on C1.1 cells. This PLA detects the interaction of two proteins with the appearance of red/ green fluorescent spots at the location of protein-protein interaction. In this case, $\beta$-catenin interacted with either Foxo (red spot) or TCF (green spot).

As shown in Figure 1B, combined treatment of TGF- $\beta$ with either ICG-001 or iCRT3 ( $\beta$-catenin/TCF inhibitors) in C1.1 cells significantly increased $\beta$-catenin/Foxo interactions (red spots) (Figure 1C, for area defined by Figure 1A) compared with the vehicle or TGF- $\beta$ alone.

Treatment with TGF- $\beta$ alone increased $\beta$-catenin/TCF interactions (green spots) compared with control. However, treatment with TGF- $\beta$ combined with either ICG-001 or iCRT3 significantly reduced $\beta$-catenin/TCF interactions (Figure 1, B and D).

These results demonstrate that TGF- $\beta$ with either ICG001 or iCRT3 increased $\beta$-catenin/Foxo interaction, most likely through inhibition of $\beta$-catenin/TCF interaction, thereby shifting $\beta$-catenin binding from TCF to Foxo.

\section{$\beta$-Catenin/Foxo Promotes Epithelial Marker Expression in Scratch Assay}

To determine the role of $\beta$-catenin/Foxo in epithelial cell differentiation, E-cadherin (epithelial marker) and $\alpha$-SMA (mesenchymal marker) dual staining was performed in C1.1 cells in the scratch assay.

After 48 hours of rhTGF- $\beta$ treatment, E-cadherin expression decreased, whereas $\alpha$-SMA expression increased, in the C1.1 cells treated, showing epithelial-tomesenchymal differentiation (Figure 2). In contrast, treatment with TGF- $\beta$ combined with either ICG-001 or iCRT3 showed dominant epithelial cell [E-cadherin $(+) \alpha$-SMA $(-)]$ in the scratched area compared with treatment with TGF- $\beta$ alone (Figure 2). In addition to ICG-00 and iCRT3 to inhibit $\beta$-catenin/TCF, a known Foxo activator, epigallocatechin 3 -gallate, ${ }^{24}$ was used to directly increase $\beta$ catenin/Foxo. Epigallocatechin 3-gallate treatment of C1.1 cells also prevented the rhTGF- $\beta$-induced down-regulation of E-cadherin and the increase of $\alpha$-SMA, providing direct evidence for $\beta$-catenin/Foxo in preventing rhTGF$\beta$-induced mesenchymal differentiation of $C 1.1$ cells.

These results demonstrate that TGF- $\beta$-induced mesenchymal differentiation is inhibited by either inhibition of $\beta$ catenin/TCF or direct activation of $\beta$-catenin/Foxo.

\section{Foxo1 Deletion Delays Wound Closure in C1.1 Cells}

To further investigate the direct role of Foxo in wound healing, Foxol and TCF1 were knocked out in C1.1 cells

\footnotetext{
Figure 3 In vitro scratch assay in wild-type (WT), Foxo1 knockout (K0), and TCF1 KO C1.1 cells. A: Representative images of the wound closure of WT, Foxo1 K0, and TCF1 K0 C1.1 untreated cells (control) or cells treated with recombinant human transforming growth factor- $\beta 1$ (rhTGF- $\beta 1$ ), rhTGF- $\beta 1$ and ICG-001, rhTGF- $\beta 1$ and iCRT3, ICG-001 alone, or iCRT3 alone. White dotted lines indicate front edge of cells after scratch at 0 hour. B and C: Quantitation of percentage wound closure (B) and number of cells (C) at 48 hours in WT (black dots), Foxo1 K0 (reds dots), and TCF1 K0 (green dots) with various treatments is shown. Results are representative of four independent experiments. Statistical significance was determined by one-way analysis of variance, followed by Tukey post hoc test. Results are shown as means \pm SEM (B and $\mathbf{C}) .{ }^{*} P<0.05,{ }^{*} P<0.01$, and ${ }^{* * *} P<0.001$. Scale bar $=100 \mu \mathrm{m}(\mathbf{A})$. Original magnification, $\times 20(A)$.
} 
WT

\section{Hours}

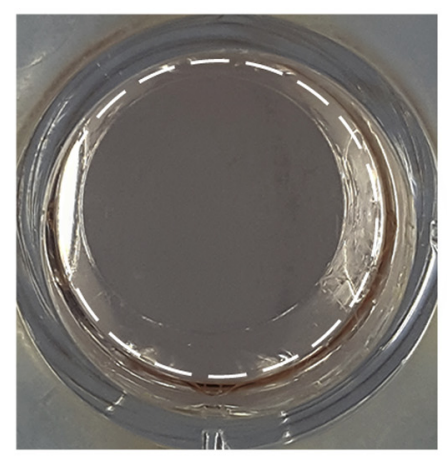

\section{Hours}

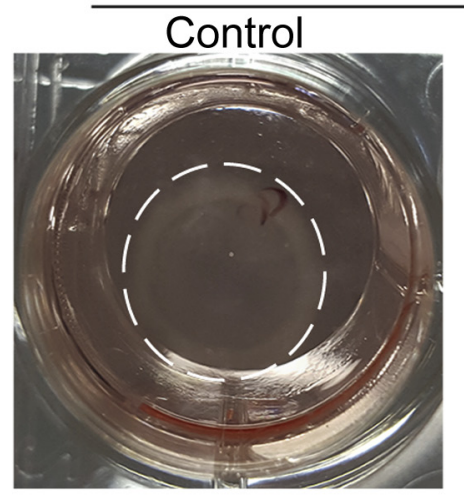

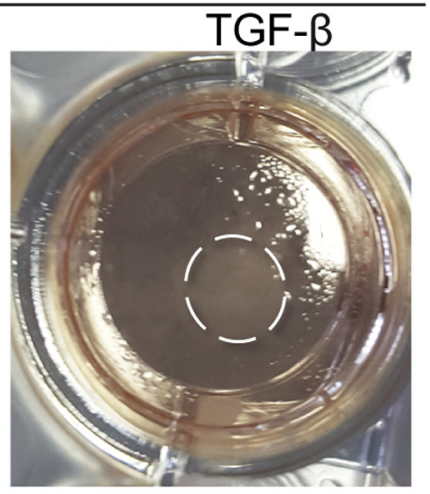

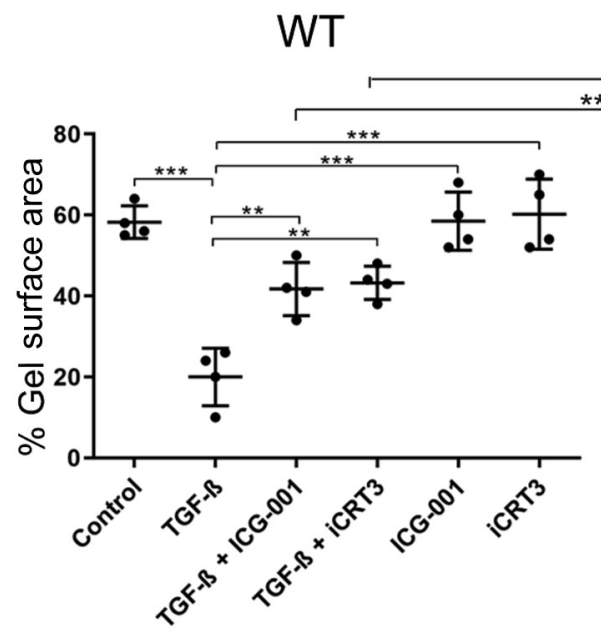

Foxo1 KO

TCF1 KO
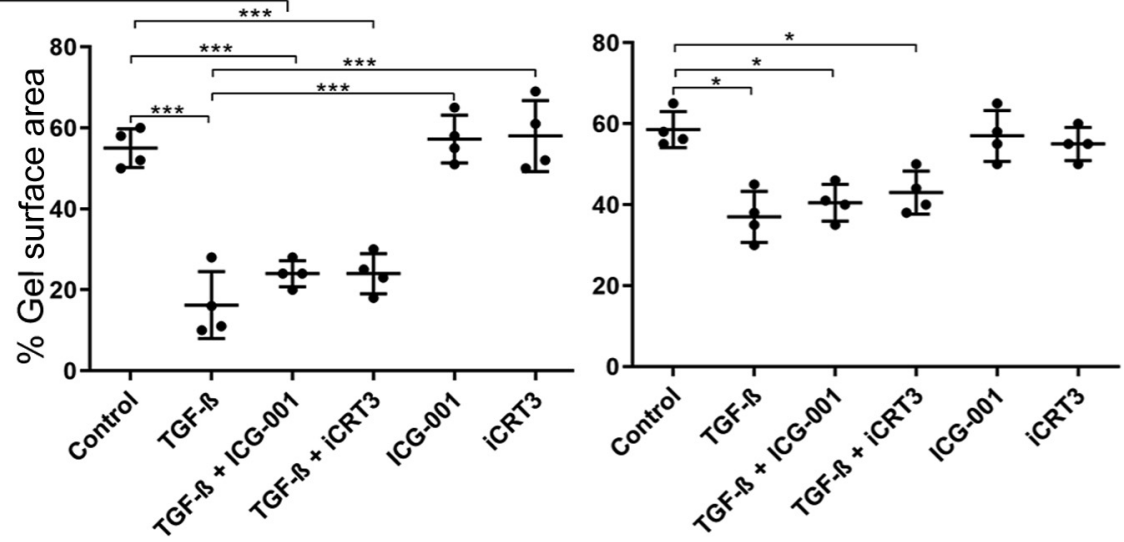

Figure 5 In vitro collagen gel contraction assay in wild-type (WT), Foxo1 knockout (K0), and TCF1 KO C1.1 cells. Top panels: Representative images of a collagen gel in a well from the bottom of plate. White dotted circle lines indicate edge of collagen gel at 0 hour. Bottom panels: Quantitation of percentage gel surface area at 48 hours in WT, Foxo1 K0, and TCF1 KO with various treatments. Results are representative of four independent experiments. Statistical significance was determined by one-way analysis of variance, followed by Tukey post hoc test. Results are shown as means \pm SEM (bottom panels). ${ }^{\star} P<0.05$, ${ }^{* *} P<0.01$, and ${ }^{* *} P<0.001$. TGF- $\beta$, transforming growth factor- $\beta$.

using CRISPR/Cas9 KO plasmids ${ }^{15}$ and wound closure was assessed using the in vitro scratch assay.

As expected, TGF- $\beta$-treated wild-type (WT) C1.1 cells showed higher capacity to close the wound than control C1.1 cells (Figure 1A, Supplemental Figure S1). Interestingly, treatment with rhTGF- $\beta 1$ combined with ICG-001 or iCRT3 increased closure of the wound compared with ICG001 or iCRT3 alone (Figure 3).

Foxol KO in C1.1 cells (Figure 3) significantly reduced closure of the wound gap in control KO cells compared with WT control cells, showing a dependence on Foxo1. The migration of rhTGF- $\beta 1$-treated Foxol KO C1.1cells was significantly slower than that of WT C1.1 cells, consistent with a role for Foxo1 in TGF- $\beta 1$-induced wound closure. More important, treatment with either ICG-001 or iCRT3 alone in Foxol KO C1.1 cells showed significantly slower migration than control TCF1 KO C1.1 cells (Figure 3). ICG-
001 or iCRT3 alone treatment, which augments $\beta$-catenin/ Foxo1, also reduced closure (Figure $3 \mathrm{~B}$ ), which may be interpreted as a result of ICG-001 or iCRT3 inhibition of cell proliferation (Figure 3C). These results suggest that $\beta$ catenin/Foxo1 is involved in the $\beta$-catenin/TCF-independent wound closure.

As expected, TCF1 KO in C1.1 cells slowed the wound closure in both control and rhTGF- $\beta 1$ treatment groups (Figure 3). However, Foxol KO in C1.1 cells further slowed the wound closure in comparison with TCF1 KO. This result suggests that the slowed wound closure in Foxol KO C1.1 cells may also be interpreted as being a result of absence of $\beta$-catenin/Foxo interaction when $\beta$-catenin/TCF binding was inhibited. Previous PLA data ${ }^{21}$ show that the combined treatment of rhTGF- $\beta$ and ICG-001 or iCRT3 greatly increased $\beta$-catenin/Foxo interaction, suggesting a role for $\beta$-catenin/Foxo interaction in wound closure. 
A

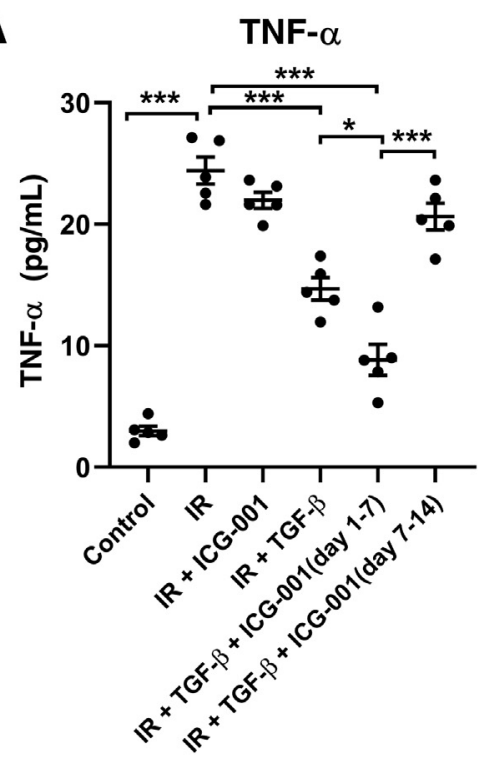

C

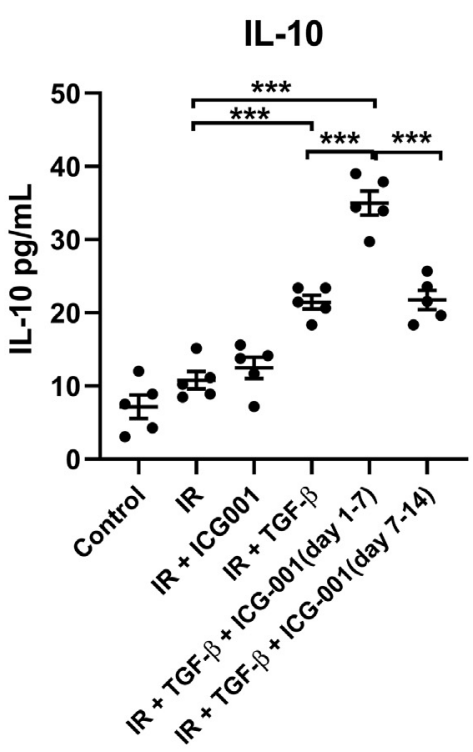

B

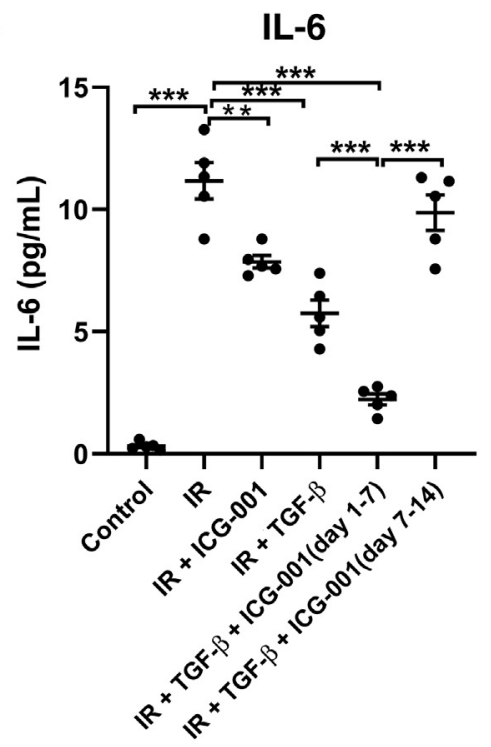

D

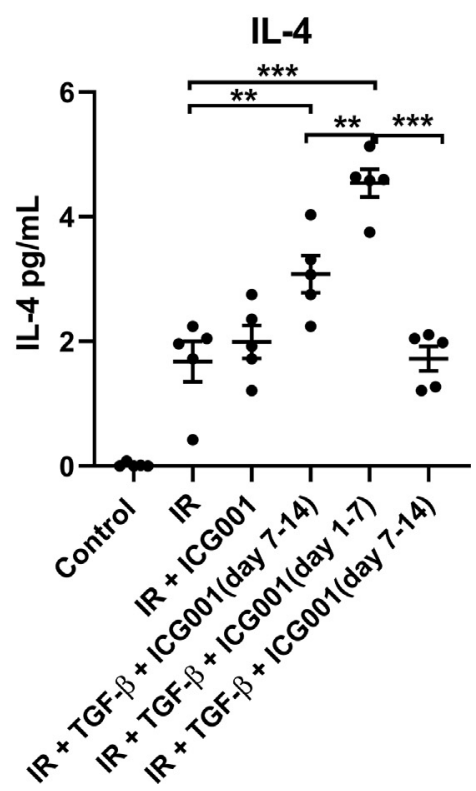

Figure 6 Inflammatory [tumor necrosis factor (TNF)- $\alpha$ and IL-6] and anti-inflammatory (IL-10 and IL-4) cytokine levels in kidney of unilateral ischemia reperfusion (IR) mice. Quantitation of inflammatory cytokines TNF- $\alpha$ (A) and IL- 6 (B) and anti-inflammatory cytokines IL-10 (C) and IL-4 (D) levels, analyzed using cytokine cytometric bead array. Statistical significance was determined by one-way analysis of variance, followed by Tukey post hoc test. Results are shown as means \pm SEM $(\mathbf{A}-\mathbf{D}) . n=5$ (A-D). ${ }^{*} P<0.05,{ }^{*} P<0.01$, and $* * * P<0.001$. TGF- $\beta$, transforming growth factor- $\beta$.
ICG-001 or iCRT3 Inhibits TGF- $\beta 1$-Induced Collagen Expression in Scratch Assay

Collagen is a key component in wound healing and fibrosis. Sirius red collagen detection assay was performed to determine whether promotion of $\beta$-catenin/Foxo interaction by using inhibitor ICG-001 or iCRT3 alters TGF$\beta$-induced collagen in the in vitro scratch assay.

Significantly increased collagen expression was observed in TGF- $\beta$-treated WT C1. 1 cells as compared to that in WT untreated (control) cells (Figure 4). Treatment with rhTGF- $\beta 1$ combined with ICG-001 or iCRT3 significantly reduced collagen expression compared with that in rhTGF$\beta 1$-treated C1.1 cells (Figure 4). However, this reduction by the combined treatment was abolished in the absence of
Foxo1 in Foxo1 KO C1.1 cells (Figure 4), indicating a dependence on Foxo1. Collagen expression levels were generally higher in all groups of Foxo1 KO cells than in those of WT, demonstrating an inhibitory role for Foxo1.

To investigate whether the inhibitory role for Foxol on collagen expression involves $\beta$-catenin/Foxo1 interaction, a targeted degradation of cytosolic $\beta$-catenin by $\beta$ TrCP-Ecadherin chimera (F-TrCP-Ecad chimera) construct was performed. ${ }^{25}$ Foxol KO C1.1 and TCF1 KO C1.1 cells were transiently transfected with F-TrCP-Ecad plasmid (Figure 4). In Foxol KO C1.1 cells, TGF- $\beta 1-$ induced collagen expression was reduced by F-TrCP-Ecad transfection, similar to that of ICG-001 or iCRT3 treatment (Figure 4), showing a $\beta$-catenin-dependent profibrotic effect generally believed to be through $\beta$-catenin/TCF. 
A

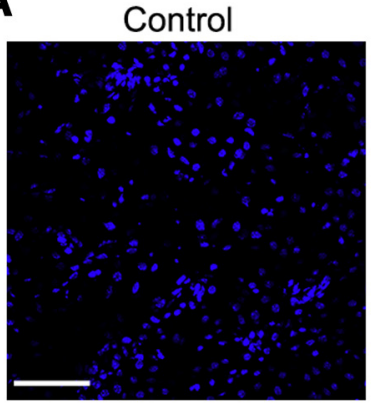

IR + rhTGF- $\beta 1$

(day 1 and day 7 )

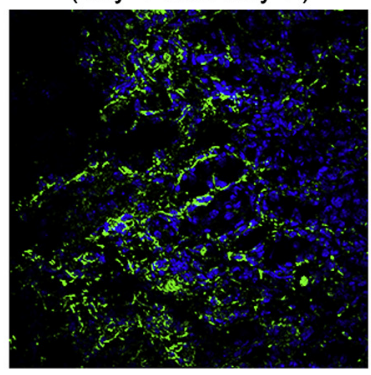

IR

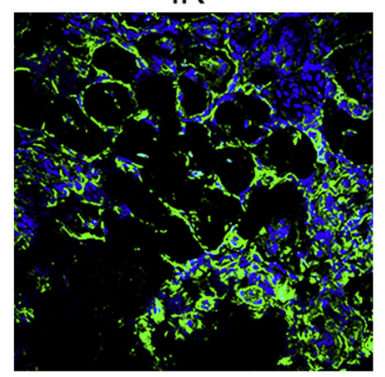

$\mathrm{IR}+\operatorname{rhTGF}-\beta 1+\mathrm{ICG}-001$ (day 1 and day 7 )

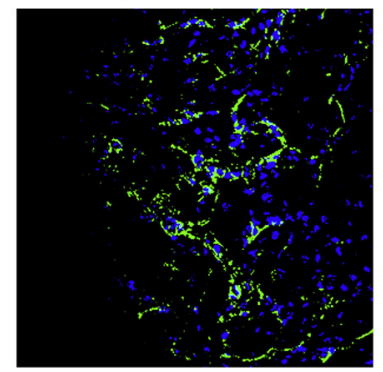

IR + ICG-001

(day 1 and day 7)

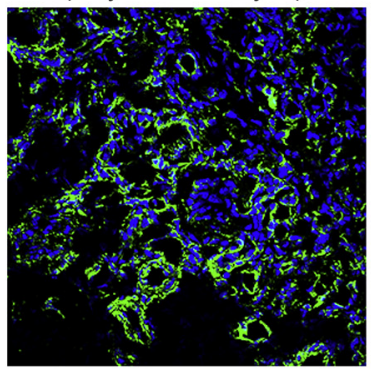

IR + rhTGF- $\beta 1+$ ICG-001 (day 1 and day 7 )

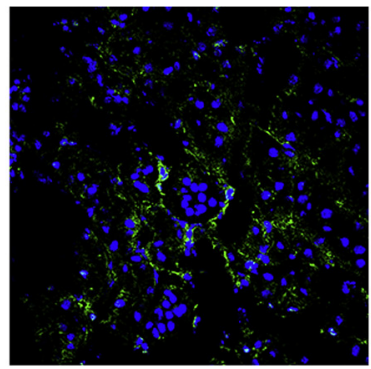

B

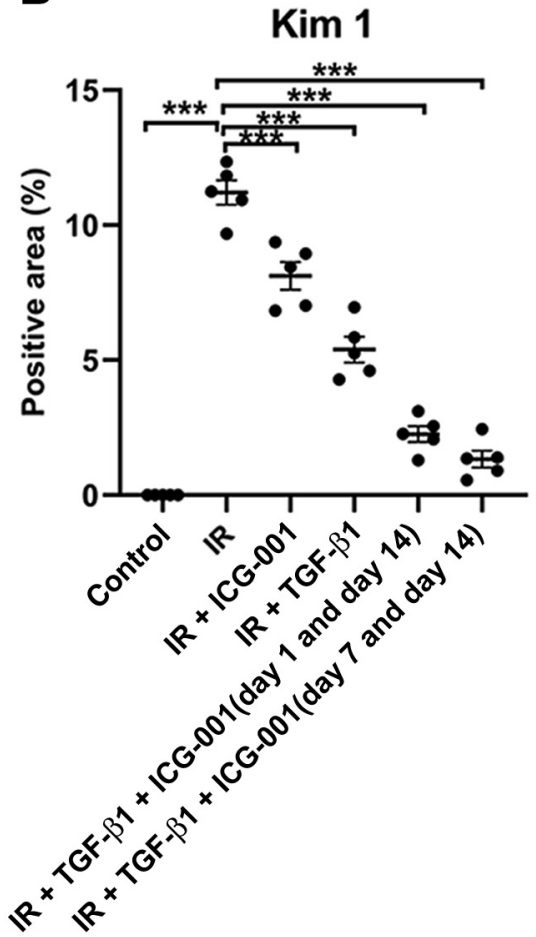

Figure 7 Kidney injury molecule 1 (Kim 1) expression in kidney of unilateral ischemia reperfusion (UIR) mice. A: Representative immunofluorescence images of Kim 1 (green) staining of kidney sections of control, untreated UIR (IR), and UIR mice treated with recombinant human transforming growth factor$\beta 1$ (rhTGF- $\beta 1$ ), rhTGF- $\beta 1$ plus ICG-001 (day 1 to 7), rhTGF- $\beta 1$ plus ICG-001 (day 7 to 14 ), or ICG-001 alone. Nuclei were stained with DAPI. B: Quantitation of positive Kim 1 (green) staining area of kidney sections relative to the total area analyzed and compared for various treatments by one-way analysis of variance, followed by Tukey post hoc test. Results are shown as means \pm SEM (B). $n=5$ (B). ${ }^{* * *} p<0.001$. Scale bar $=50 \mu \mathrm{m}(\mathbf{A})$.

$\beta$-Catenin is known to bind to either TCF or Foxo in competition. ${ }^{26}$ In TCF KO C1.1 cells, $\beta$-catenin uses Foxo as its binding partner. However, degradation of $\beta$-catenin by F-TrCP-Ecad increased rhTGF- $\beta 1$-induced collagen in TCF1 KO C1.1 cells, demonstrating an inhibitory role for $\beta$ catenin in absence of TCF in rhTGF- $\beta 1$-induced collagen expression. This result suggested that $\beta$-catenin binding to Foxo has a direct negative effect on collagen expression, which is $\beta$-catenin/TCF independent in the TCF KO cells. This notion was supported by the fact that the combined treatment resulted in further reduction of collagen expression in WT (Figure 4) as compared to that in TCF KO cells (Figure 4), which can be explained by shifting of $\beta$-catenin from TCF to Foxo1 in WT cells, not seen in either Foxo or $T C F$ KO. This result shows that $\beta$-catenin binding to Foxo is required to reduce rhTGF- $\beta 1$-induced collagen expression in the in vitro scratch assay. To study further the effect on collagen, the contraction of wound healing was examined via collagen gel contraction assay.

\section{ICG-001 or iCRT3 Inhibits TGF- $\beta 1$-Induced Contraction in Collagen Gel Contraction Assay}

TGF- $\beta$-induced $\alpha$-SMA expression is responsible for fibroblast-mediated contraction in wound healing. An in vitro collagen gel contraction assay was performed to determine whether ICG-001 or iCRT3 inhibits TGF$\beta$-induced collagen gel contraction. Contractile activity was analyzed by measuring the area of contracted collagen gel and expressed as a percentage of the original area. Although this result may not apply to tubular cell injury repair, it is used to assess whether catenin/Foxo1 reduced collagen expression leading to a substantial negative impact on wound closure.

As shown in Figure 5, TGF- $\beta$ induced strong contraction of collagen and reduced the gel surface area in WT C1.1 cells compared with that in control at 48 hours. Treatment with TGF- $\beta$ combined with either ICG-001 or iCRT3 significantly inhibited the TGF- $\beta$-induced contraction of collagen gel (Figure 5), indicating a role for $\beta$-catenin/TCF in TGF- $\beta$-induced contraction of collagen gel. Compared with control, there was little, if any, change in contraction of collagen gel surface area in WT C1.1 cells treated with ICG001 or iCRT3 alone.

TGF- $\beta$ induced stronger collagen gel contraction in Foxol KO in C1.1 cells (Figure 5) as compared to that in WT cells. In addition, the combined treatment of TGF- $\beta$ and ICG001 or iCTR3 also displayed stronger TGF$\beta$-induced collagen gel contraction in Foxol KO C1.1 cells compared with that of WT C1.1 cells, showing 


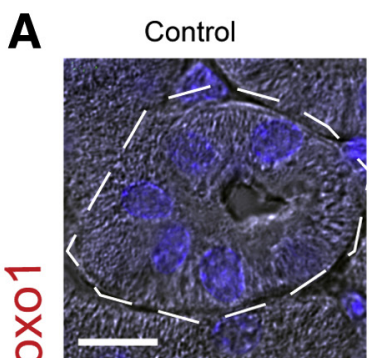

IR + rhTGF- $\beta 1$

(day 1-7)

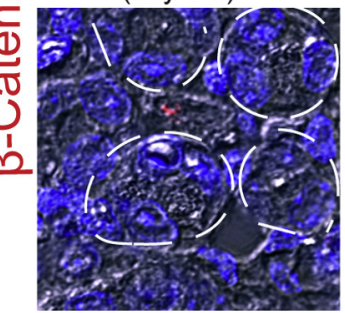

B

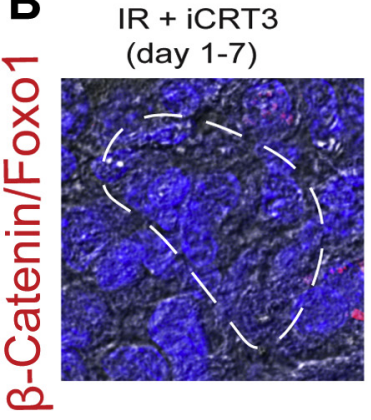

IR

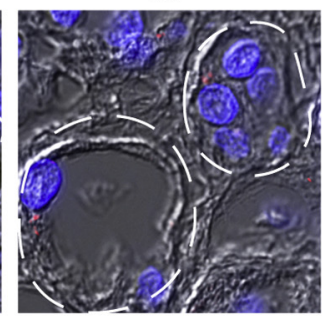

IR + rhTGF- $\beta 1+$ ICG-001 (day 1-7)

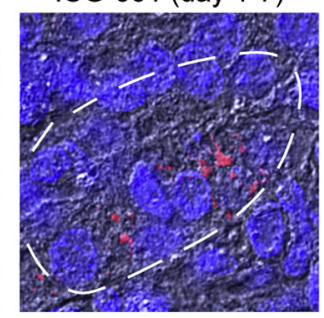

$\mathrm{IR}+$ rhTGF- $\beta 1+\mathrm{iCRT3}$ (day 1-7)

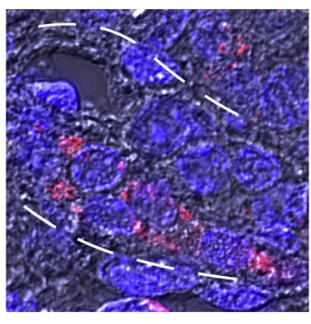

$\mathrm{IR}+\mathrm{ICG}-001$

(day 1-7)

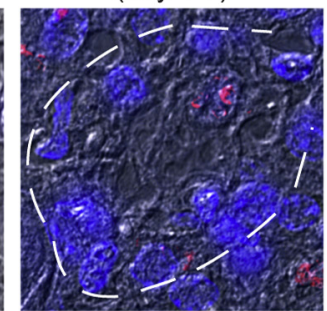

IR + rhTGF- $\beta 1+$ ICG-001 (day 7-14)
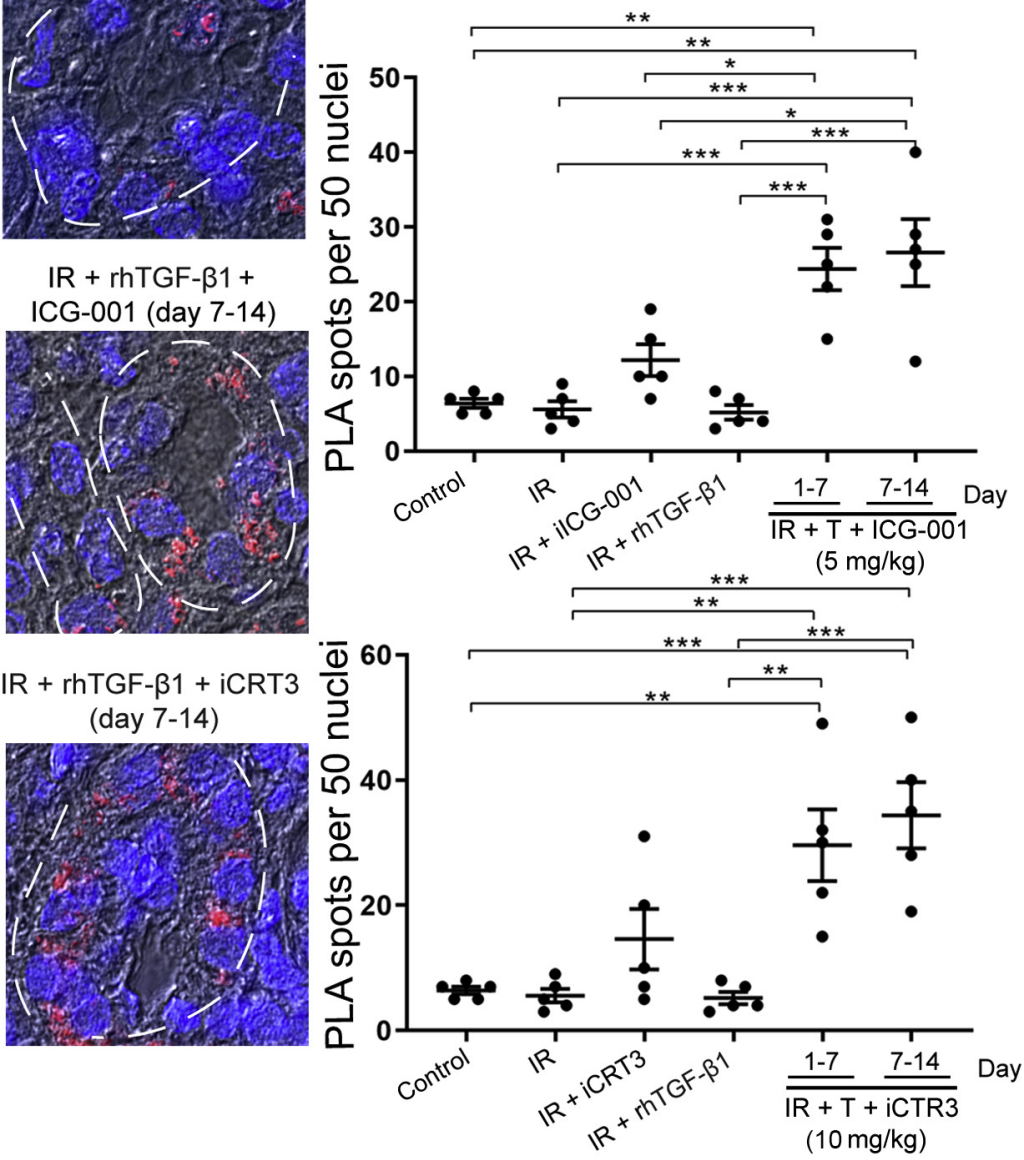

Figure 8 Proximity ligation assay (PLA) analysis of $\beta$-catenin/forkhead box protein 01 (Foxo1) interactions in kidney of unilateral ischemia reperfusion (UIR) mice. A: Representative $\beta$-catenin/Foxo1 (red spot) interaction in kidney sections of control, untreated UIR, and UIR mice treated with recombinant human transforming growth factor- $\beta 1$ (rhTGF- $\beta 1$ ), rhTGF- $\beta 1$ and ICG-001 (day 1 to 7), rhTGF- $\beta 1$ and ICG-001 (day 7 to 14 ), or ICG-001 alone. B: Representative $\beta$-catenin/Fox01 (red spot) interaction in kidney sections of UIR mice treated with iCRT3 alone, rhTGF- $\beta 1$ and iCRT3 (day 1 to 7 ), or rhTGF- $\beta 1$ and iCRT3 (day 7 to 14). Nuclei were stained with DAPI. Fluorescence images were merged with differential interference contrast images to show the localization of PLA signals in tubular cells (indicated by broken lines). Quantitation of PLA signals with the various treatments was compared by one-way analysis of variance, followed by Tukey post hoc test. Results are shown as means $\pm \operatorname{SEM}(\mathbf{A}$ and $\mathbf{B}) . n=5(\mathbf{A}$ and $\mathbf{B}) .{ }^{*} P<0.05,{ }^{* *} P<0.01$, and ${ }^{* * *} P<0.001$. Scale bar $=40 \mu \mathrm{m}(\mathbf{A}$ and $\mathbf{B})$. Original magnification, $\times 60(\mathbf{A}$ and $\mathbf{B})$.

negative role for Foxo1. The lower reduction by ICG-001 or iCRT3 only treated Foxol KO cells than that of WT indicates an underlying role for $\beta$-catenin/Foxo1 absent from the Foxol KO cells. Together with the PLA results showing that the combined treatment shifted $\beta$-catenin from TCF to Foxo1 binding (Figure 1B), this result suggests that the reduced TGF- $\beta$-induced contraction depends on $\beta$-catenin/Foxo1.

In TCF1 KO C1.1 cells (Figure 5), TGF- $\beta$-induced collagen gel contraction was weaker than that of Foxol KO or WT C1.1 cells, demonstrating a positive role for TCF in the gel contraction. As expected, in absence of TCF, addition of ICG-001 or iCRT3 showed no difference in collagen gel contraction to that of TGF- $\beta$ only treatment (Figure 5). This shows a $\beta$-catenin/TCF dependence in TGF$\beta$-mediated collagen gel contraction.
Treatment with rhTGF- $\beta$ Combined with ICG-001 Reduces Tubular Epithelial Injury in UIR

To test in vivo for the role of $\beta$-catenin/Foxo in protection of tubular injury and promotion of epithelial repair, a kidney UIR injury model was subjected to inflammation at an early phase and injury repair at a late phase. In this injury model, C57BL $/ 6$ mice were treated with rhTGF- $\beta 1$ at $50 \mu \mathrm{g} / \mathrm{kg}$ body weight every 2 days and ICG-001 $(5 \mathrm{mg} / \mathrm{kg})$ or iCRT3 $(10 \mathrm{mg} / \mathrm{kg})$ daily from day 1 to 7 or from day 7 to 14 . Promotion of $\beta$-catenin/Foxo by TGF- $\beta$ combined with ICG-001 enhances the anti-inflammatory function of TGF- $\beta$ via up-regulation of regulatory $\mathrm{T}$ cells. ${ }^{21}$ To examine whether $\beta$-catenin/Foxo has a direct effect in promoting epithelial repair, rather than an indirect effect from reducing inflammation, the treatment group of rhTGF- $\beta 1$ combined 

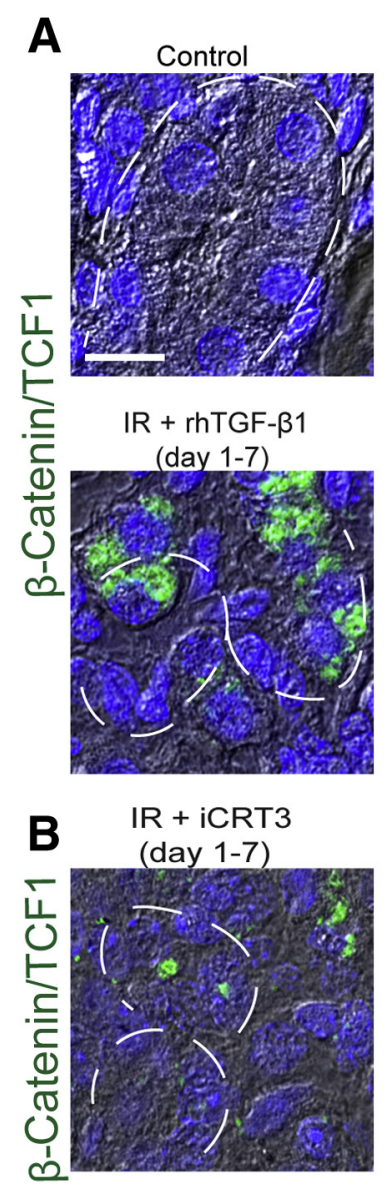
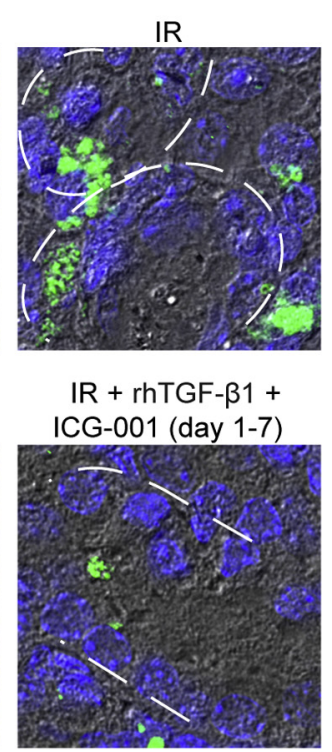

$\mathrm{IR}+$ rhTGF- $\beta 1+\mathrm{iCRT3}$ (day 1-7)

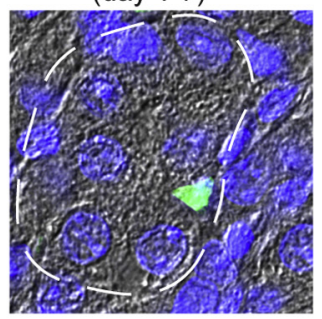

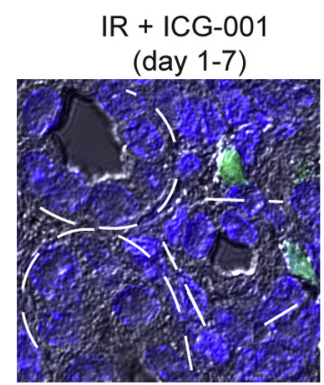

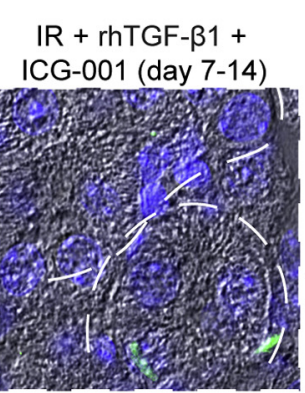

$\mathrm{IR}+$ rhTGF- $\beta 1+\mathrm{iCRT3}$

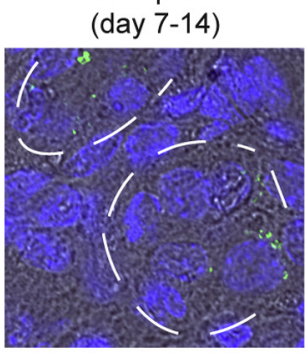

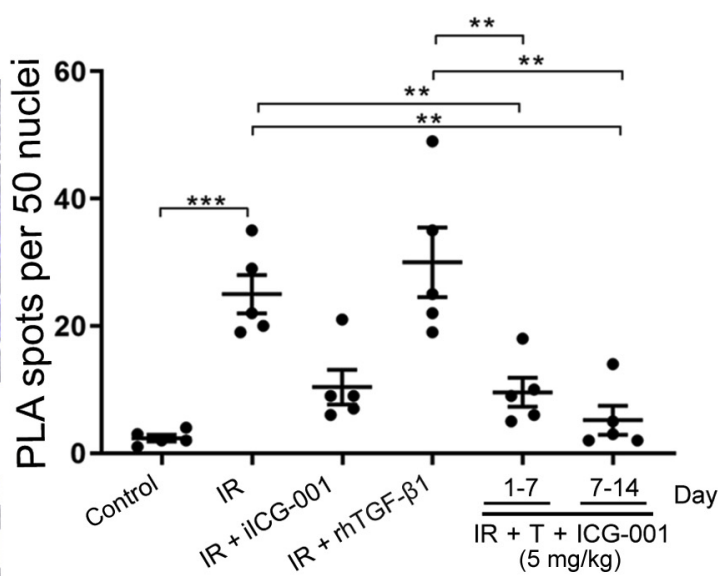
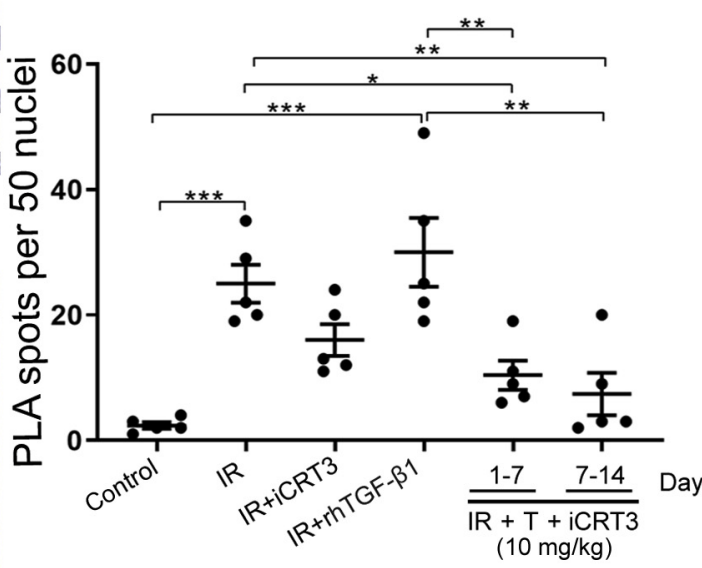

Figure 9 Proximity ligation assay (PLA) analysis of $\beta$-catenin/T-cell factor (TCF) interactions in kidney of unilateral ischemia reperfusion (UIR). A: Representative $\beta$-catenin/TCF (green spot) interaction in kidney sections of control, untreated UIR, and UIR mice treated with recombinant human transforming growth factor- $\beta 1$ (rhTGF- $\beta 1$ ), rhTGF- $\beta 1$ and ICG-001 (day 1 to 7 ), rhTGF- $\beta 1$ and ICG-001 (day 7 to 14 ), or ICG-001 alone. B: Representative $\beta$-catenin/ TCF (green spot) interaction in kidney sections of UIR mice treated with iCRT3 alone, rhTGF- $\beta 1$ and iCRT3 (day 1 to 7 ), or rhTGF- $\beta 1$ and iCRT3 (day 7 to 14 ). Nuclei were stained with DAPI. Fluorescence images were merged with differential interference contrast images to show the localization of PLA signals in tubular cells (indicated by broken lines). Quantitation of PLA signals with the various treatments was compared by one-way analysis of variance, followed by Tukey post hoc test. Results are shown as means $\pm \operatorname{SEM}(\mathbf{A}$ and $\mathbf{B}) . n=5(\mathbf{A}$ and $\mathbf{B}) .{ }^{*} P<0.05,{ }^{* *} P<0.01$, and ${ }^{* *} P<0.001$. Scale bar $=40 \mu \mathrm{m}(\mathbf{A}$ and $\mathbf{B})$. Original magnification, $\times 60(\mathbf{A}$ and $\mathbf{B})$.

with ICG-001 was also divided into an early (day 1 to 7) and a late (day 7 to 14) treatment group. Early days (day 1 to 7 ) after UIR are predominantly inflammatory, and the combined treatment at early days is anti-inflammatory due to an increase in regulatory $\mathrm{T}$ cells. ${ }^{21}$ In the current study, early treatment, but not late treatment, with rhTGF- $\beta$ combined with ICG-001, reduced inflammatory cytokines tumor necrosis factor- $\alpha$ and IL-6, whereas it increased antiinflammatory cytokines IL-10 and IL-4 in UIR kidney (Figure 6). This result demonstrates that the combined treatment generated an anti-inflammatory effect in early inflammation phase of UIR, but not in late phase, suggesting that any protection in late treatment is independent of the enhanced anti-inflammation function by the combined treatment.

The kidney tubular injury was assessed by immunofluorescence staining of the kidney tubular injury marker, kidney injury molecule 1 (Kim 1), ${ }^{27,28}$ in UIR kidney. Ischemia reperfusion injury significantly increased Kim 1 expression in UIR kidney.
However, both ICG-001- or rhTGF- $\beta 1$-only treatment in early phase reduced Kim 1 expression (Figure 7), indicating protection of tubular injury, given anti-inflammatory effects by rhTGF- $\beta 1^{21}$ and antifibrotic function by ICG- $001^{15,20}$ as observed by us and others. More importantly, the combined treatment by rhTGF- $\beta 1$ and ICG-001 in both early and late phases further reduced Kim 1 expression levels in UIR kidney, demonstrating protective effects by promotion of $\beta$-catenin/ Foxo. Given a predominant anti-inflammatory effect by the early treatment, the late treatment showed an anti-inflammatoryindependent protection.

Redirection of $\beta$-Catenin Binding from TCF to Foxo by rhTGF- $\beta$ Combined with Either ICG-001 or iCRT3 in UIR

As a protective effect of combined treatment with rhTGF- $\beta$ and ICG-001 or rhTGF- $\beta$ and iCRT3 was observed in UIR mice, whether $\beta$-catenin binding shifts from TCF to Foxo in vivo by PLA in kidney of UIR mice was investigated. Co- 


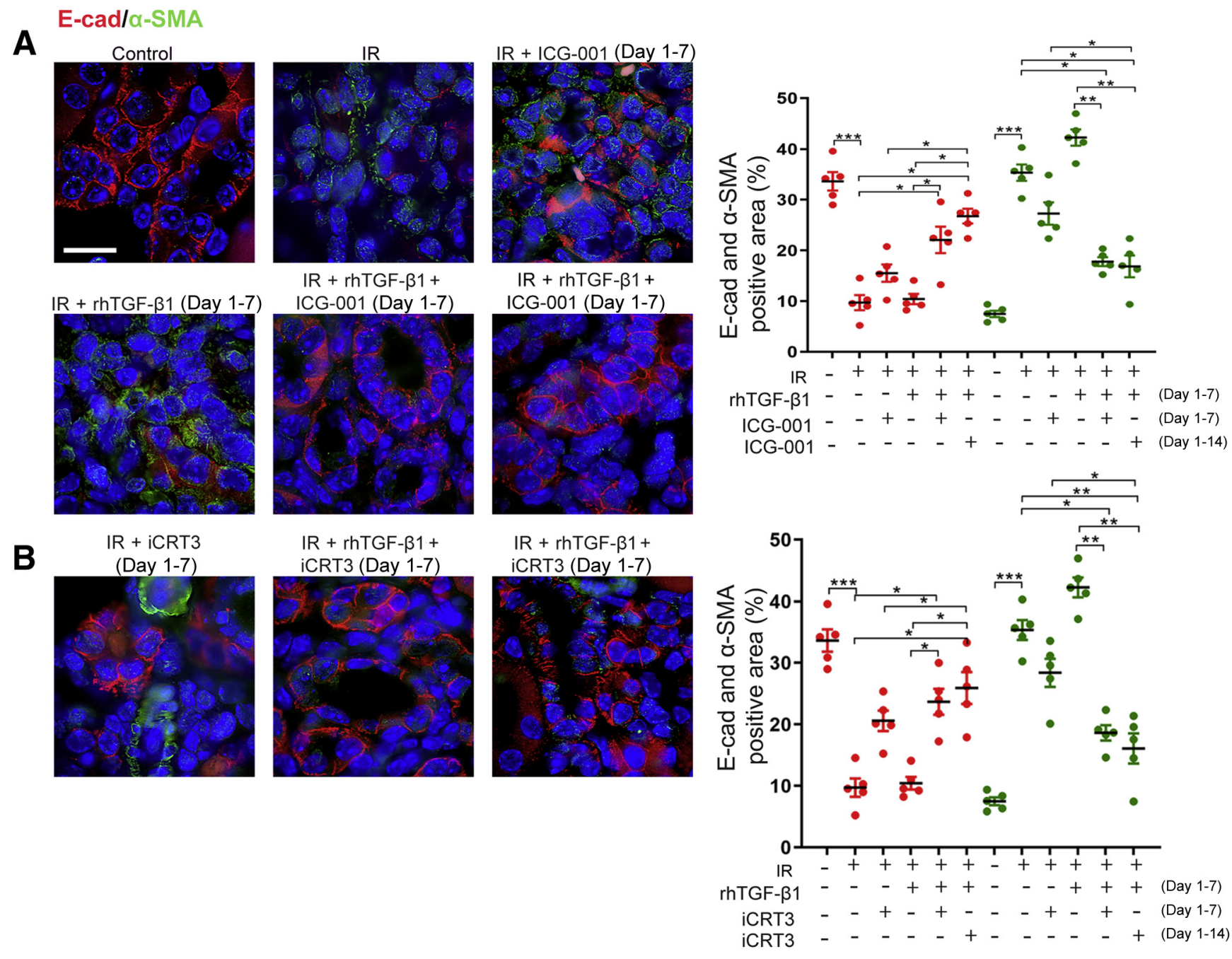

Figure 10 E-cadherin (E-cad) and $\alpha$-smooth muscle actin ( $\alpha$-SMA) expression in kidney of unilateral ischemia reperfusion (UIR) mice. A: Representative immunofluorescence images of E-cadherin (red) and $\alpha$-SMA (green) staining of kidney sections of control, untreated UIR, and UIR mice treated with recombinant human transforming growth factor- $\beta 1$ (rhTGF- $\beta 1$ ), rhTGF- $\beta 1$ plus ICG-001 (day 1 to 7), rhTGF- $\beta 1$ plus ICG-001 (day 7 to 14 ), or ICG-001 alone. B: Representative immunofluorescence images of E-cadherin (red) and $\alpha$-SMA (green) staining of kidney sections of UIR mice treated with iCRT3 alone, rhTGF- $\beta 1$ plus iCRT3 (day 1 to 7), or rhTGF- $\beta 1$ plus iCRT3 (day 7 to 14). Nuclei were stained with DAPI. Quantitation of positive E-cadherin (red) and $\alpha$-SMA (green) staining area of kidney sections relative to the total area from 10 random microscopic fields was analyzed and compared for various treatments by one-way analysis of variance, followed by Tukey post hoc test. Results are shown as means \pm SEM (A and $\mathbf{B}) . n=5(\mathbf{A}$ and $\mathbf{B})$. ${ }^{*} P<0.05,{ }^{* *} P<0.01$, and $* * * P<0.001$. Scale bar $=40 \mu \mathrm{m}(\mathbf{A}$ and $\mathbf{B})$. Original magnification, $\times 60(\mathbf{A}$ and $\mathbf{B})$.

administration of rhTGF- $\beta$ with either ICG-001 or iCRT3 showed significantly greater $\beta$-catenin/Foxo (red) interactions (Figure 8) than in UIR mice treated with TGF- $\beta$ alone. Similar to early treatment, late administration of rhTGF- $\beta$ with either ICG-001 or iCRT3 also showed significantly greater $\beta$-catenin/Foxo interactions than in UIR mice treated with TGF- $\beta$ alone. Treatment with either ICG001 or iCRT3 alone showed increased $\beta$-catenin/Foxo interactions compared with vehicle or TGF- $\beta$-treated UIR mice but significantly less than the combined treatment of rhTGF- $\beta$ with either ICG-001 or iCRT3 (Figure 8).

As expected, $\beta$-catenin/TCF (green) signal was significantly increased in UIR mice, whereas rhTGF- $\beta$ treatment further increased $\beta$-catenin/TCF interaction compared with control kidney (Figure 9). Interestingly, in UIR mice, $\beta$ -
catenin/TCF interaction was significantly reduced with coadministration of rhTGF- $\beta 1$ with either ICG-001 or iCRT3 by both early and late treatment compared with TGF- $\beta$-treated UIR mice (Figure 9).

These in vivo results show direct evidence for shifting of $\beta$-catenin binding from TCF to Foxo following combined treatment of rhTGF- $\beta$ with either ICG-001 or iCRT3. These results are similar to those obtained in the in vitro PLA study.

Treatment with rhTGF- $\beta$ Combined with Either ICG-001 or iCRT3 Promotes Epithelial Repair in UIR

To assess whether fibrotic mesenchymal cells or physiological epithelial cells are used for kidney repair, 
A

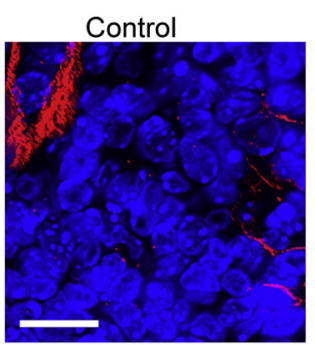

IR + rhTGF- $\beta 1$ (day 1-7)

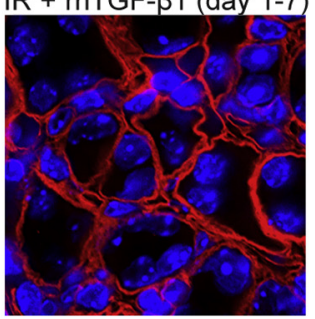

IR + iCRT3

B

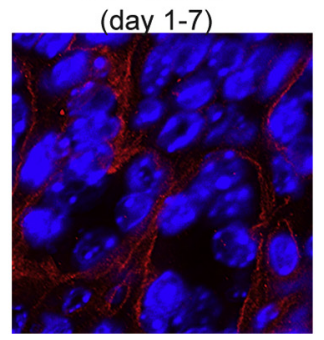

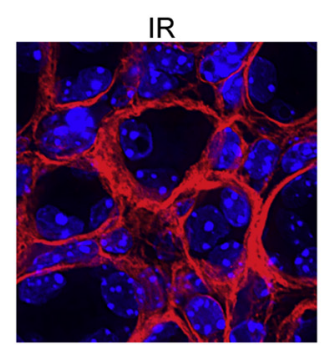

IR + rhTGF- $\beta 1$ +
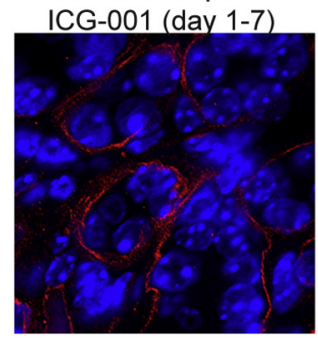

IR + rhTGF- $\beta 1+$ iCRT3 (day 1-7)

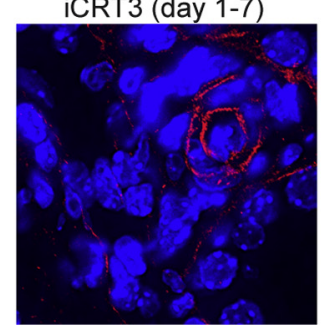

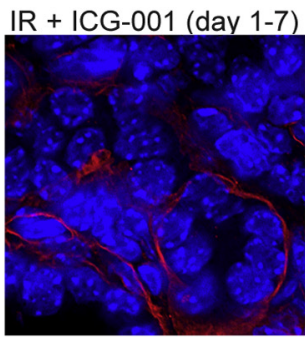

IR + rhTGF- $\beta 1+$
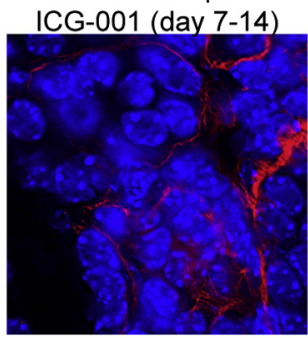

IR + rhTGF- $\beta 1+$

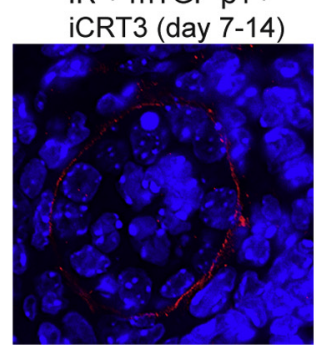

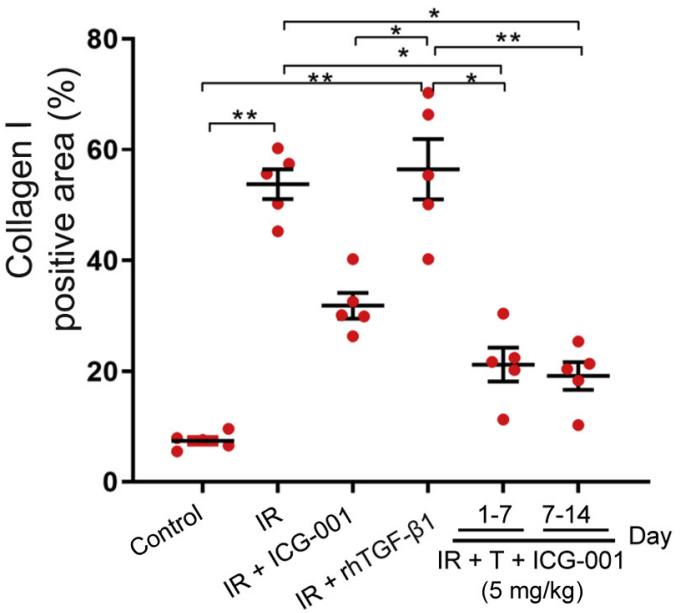
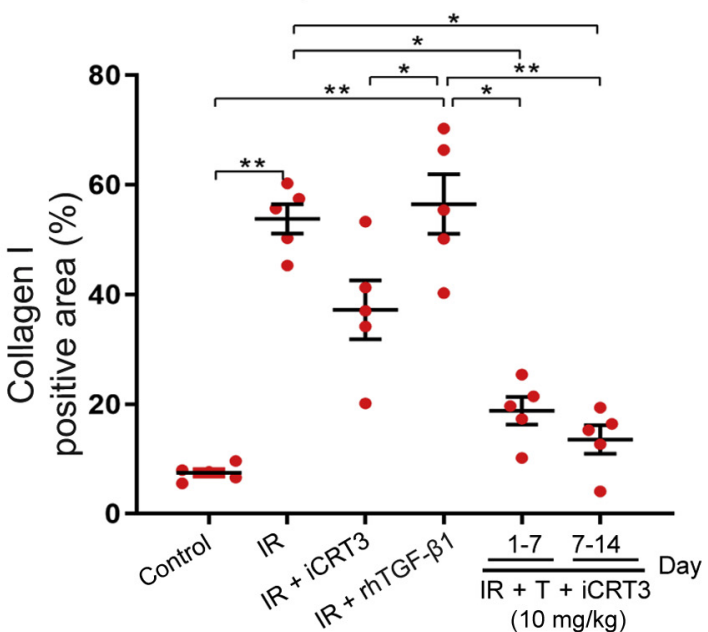

Figure 11 Collagen I expression in kidney of unilateral ischemia reperfusion (UIR) mice. A: Representative immunofluorescence images of collagen I staining of kidney sections of control, untreated UIR, and UIR mice treated with recombinant human transforming growth factor- $\beta 1$ (rhTGF- $\beta 1$ ), rhTGF- $\beta 1$ plus ICG-001 (day 1 to 7), rhTGF- $\beta 1$ plus ICG-001 (day 7 to 14), or ICG-001 alone. B: Representative immunofluorescence images of collagen I staining of kidney sections of UIR mice treated with iCRT3 alone, rhTGF- $\beta 1$ plus iCRT3 (day 1 to 7 ), or rhTGF- $\beta 1$ plus iCRT3 (day 7 to 14). Nuclei were stained with DAPI. Quantitation of positive collagen I staining area of kidney sections relative to the total area from 10 random microscopic fields was analyzed with various treatments and compared by one-way analysis of variance, followed by Tukey post hoc test. Results are shown as means \pm SEM $(\mathbf{A}$ and $\mathbf{B}) . n=5(\mathbf{A}$ and $\mathbf{B})$. ${ }^{*} P<0.05,{ }^{* *} P<0.01$. Scale bar $=40 \mu \mathrm{m}(\mathbf{A}$ and $\mathbf{B})$. Original magnification, $\times 60(\mathbf{A}$ and $\mathbf{B})$.

myofibroblast marker ( $\alpha$-SMA) and epithelial marker (Ecadherin) were examined in the UIR injury model.

As shown in Figure 10, UIR mice treated with the vehicle or rhTGF- $\beta$ showed significantly increased $\alpha$-SMA (mesenchymal repair) and decreased E-cadherin expression compared with control. Treatment with either ICG-001 or iCRT3 alone decreased $\alpha$-SMA and increased epithelial Ecadherin expression compared with TGF- $\beta$-treated UIR mice (Figure 10). More importantly, treatment with rhTGF$\beta$ combined with either ICG-001 or iCRT3 inhibited rhTGF$\beta$-induced $\alpha$-SMA expression and showed dominant Ecadherin expression $[\alpha$-SMA $(-)$ E-cadherin $(+)]$ to a greater extent than seen with ICG-001 or iCRT3 alone (Figure 10). In addition, delayed administration of rhTGF- $\beta$ with either ICG-001 or iCRT3 also reduced rhTGF$\beta$-induced mesenchymal healing (Figure 10). These results are consistent with those obtained with the in vitro study.
Treatment with rhTGF- $\beta$ Combined with ICG-001 or iCRT3 Attenuates Kidney Fibrosis

Whether the epithelial repair seen with rhTGF- $\beta$ plus ICG001 or rhTGF- $\beta$ plus iCRT3 in UIR mice was associated with reduced interstitial fibrosis was further investigated. For this, collagen and Gomori trichrome staining was used to assess kidney fibrosis.

As shown in Figure 11A, UIR mice with or without rhTGF- $\beta$ had significantly increased collagen compared with control kidney. Treatment of UIR mice with either ICG-001 or iCRT3 alone reduced collagen compared with rhTGF- $\beta$-treated UIR mice. More importantly, combined treatment of rhTGF- $\beta$ with either ICG-001 (Figure 11A or iCRT3 (Figure 11B) significantly reduced collagen compared with rhTGF- $\beta$ alone in UIR mice (Figure 11)). Additionally, late co-administration of rhTGF- $\beta$ with either 

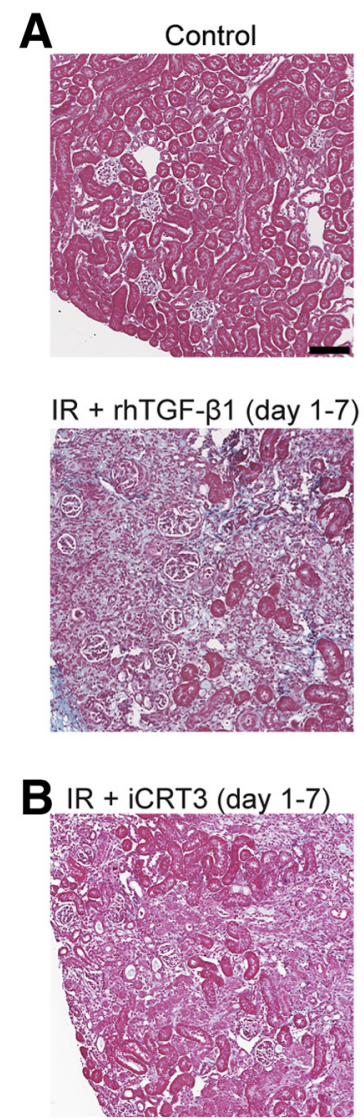

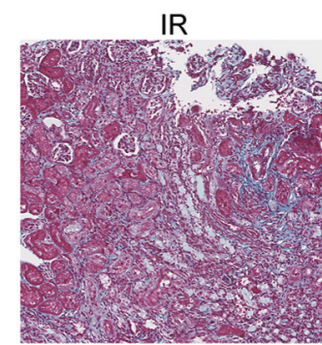

IR + rhTGF- $\beta 1+$
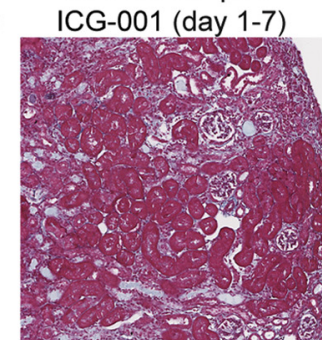

IR + rhTGF- $\beta 1+$ iCRT3 (day 1-7)

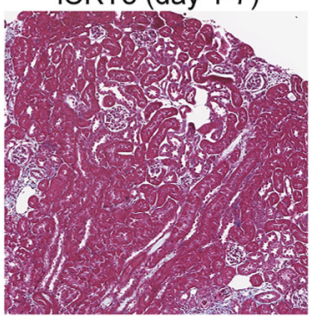

IR + ICG-001 (day 1-7)

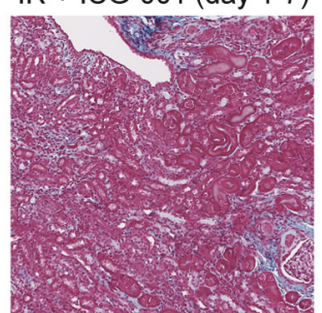

IR + rhTGF- $\beta 1$ + ICG-001 (day 7-14)

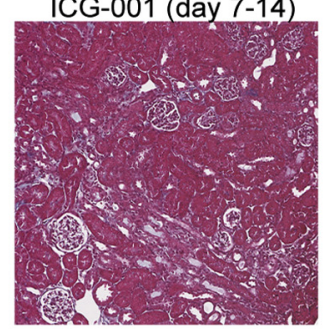

IR + rhTGF- $\beta 1$ + iCRT3 (day 7-14)

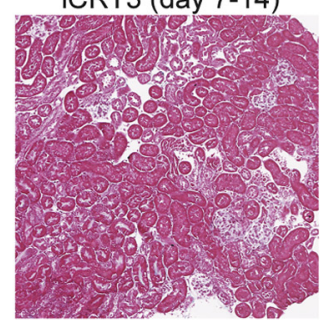

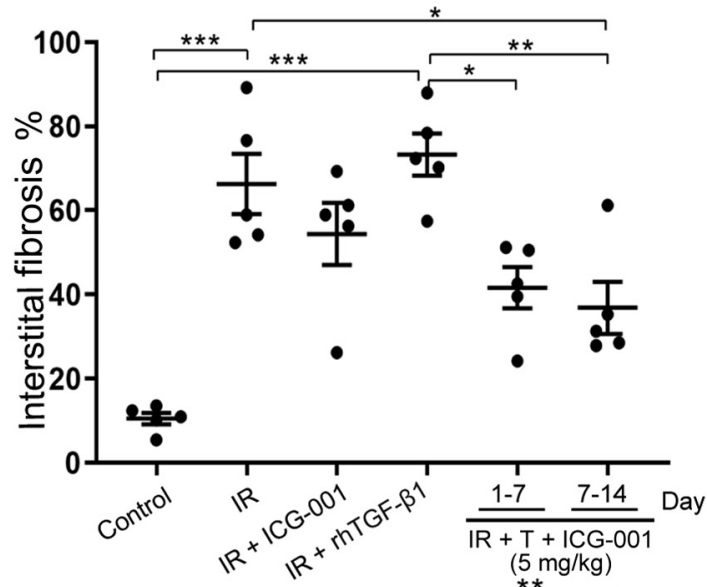

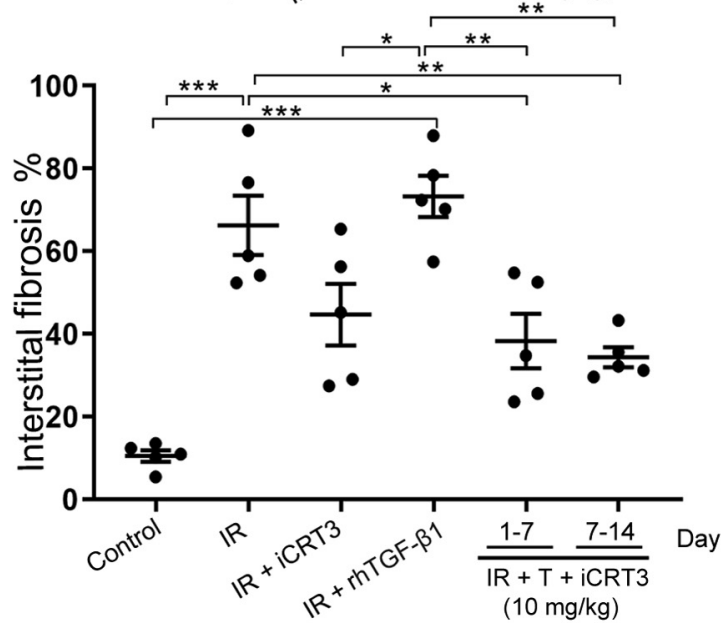

Figure 12 Kidney fibrosis in unilateral ischemia reperfusion (UIR) mice. A: Representative Gomori trichrome staining in kidney sections of control, untreated UIR, and UIR mice treated with recombinant human transforming growth factor- $\beta 1$ (rhTGF- $\beta 1$ ), rhTGF- $\beta 1$ plus ICG-001 (day 1 to 7 ), rhTGF- $\beta 1$ plus ICG-001 (day 7 to 14), or ICG-001 alone. B: Representative Gomori trichrome staining in kidney sections of UIR mice treated with iCRT3 alone, rhTGF- $\beta 1$ plus iCRT3 (day 1 to 7), or rhTGF- $\beta 1$ plus iCRT3 (day 7 to 14). Quantitation of positive tubulointerstitial area relative to the total area from 10 random microscopic fields was analyzed and compared for various treatments by one-way analysis of variance, followed by Tukey post hoc test. Results are shown as means \pm SEM (A and B). $n=5$ (A and $\mathbf{B}) .{ }^{*} P<0.05,{ }^{*} P<0.01$, and ${ }^{* * *} P<0.001$. Scale bar $=100 \mu \mathrm{m}(\mathbf{A}$ and $\mathbf{B})$. Original magnification $\times 10(\mathbf{A}$ and $\mathbf{B})$.

ICG-001 or iCRT3 significantly reduced collagen compared with rhTGF- $\beta$ alone in UIR mice.

When assessed by Gomori trichrome stain for kidney fibrosis, as shown in Figure 12A, UIR mice had significantly increased interstitial trichrome stain compared with control kidney. rhTGF- $\beta$ alone treatment also increased interstitial fibrosis compared with control kidney. Treatment of UIR mice with either ICG-001 or iCRT3 (Figure 12B) alone reduced kidney fibrosis compared with rhTGF$\beta$-treated UIR mice. Notably, combined treatment with rhTGF- $\beta$ with either ICG-001 (Figure 12A) or iCRT3 (Figure 12B) significantly attenuated the kidney fibrosis compared with rhTGF- $\beta$ alone by both early and late treatments in UIR mice (Figure 8).

Collectively, these in vivo experiments showed that treatment with rhTGF- $\beta$ combined with either ICG-001 or iCRT3 repairs the UIR kidney in a physiological epithelial repair manner with reduced collagen expression and with less fibrosis.

\section{Discussion}

Fibrosis represents a failed repair of injury with excessive ECM deposition. ${ }^{29}$ TGF- $\beta$ is an important multifunctional growth factor involved in injury repair. ${ }^{3}$ TGF- $\beta$ is known to promote healing through $\beta$-catenin/TCF. ${ }^{30}$ It activates myofibroblast (activated fibroblast) proliferation and causes excessive ECM production by myofibroblasts. Tissue that heals with excessive ECM results in fibrosis and destruction of physiological structure and function of the organ. ${ }^{31}$ Targeting $\beta$-catenin/TCF reduces fibrosis; however, whether healing could be compromised by the inhibition of $\beta$-catenin/TCF is unknown and a potential concern.

Previous studies have shown that Foxol promotes healing. ${ }^{12,13}$ Hoogeboom et $\mathrm{al}^{26}$ showed that there is reciprocal competition between TCF and Foxo in binding to $\beta$-catenin. Furthermore, Essers et $\mathrm{al}^{32}$ showed that TCF or Foxo binds to the same binding site on $\beta$-catenin. Also, our previous study showed that shifting of $\beta$-catenin binding from TCF to 
Foxo promoted TGF- $\beta^{\prime}$ s anti-inflammatory effects by upregulation of T-regulatory cells ${ }^{21}$ and plays a protective role. ${ }^{15}$ However, whether shifting of $\beta$-catenin/TCF to $\beta$ catenin/Foxo plays a role in kidney injury repair is unknown. To test this, CRISPR/Cas9-mediated knockout of Foxo or TCF was performed in vitro to show the importance of $\beta$-catenin/Foxo in TGF- $\beta$-mediated tubular epithelial cell proliferation and differentiation, collagen production, and collagen gel contraction. Also, combined treatment of TGF- $\beta$ with either ICG-001 or iCRT3 reduced kidney fibrosis in kidney injury and repair in UIR model, accompanied by increased $\beta$-catenin/Foxo1 interaction in tubules. The latter is an additional mechanism to explain the greater epithelial repair by promotion of $\beta$-catenin/Foxo interaction.

To investigate the role for $\beta$-catenin/Foxo in repair, CRISPR/Cas9 KO of Foxo or TCF was used in an in vitro scratch assay. The dependence on Foxo in TGF$\beta$-mediated epithelial cell proliferation and differentiation was shown in the Foxo knockout. TCF KO also demonstrated a role for TCF in promotion of epithelial-mesenchymal differentiation and proliferation, but less than for Foxo. Promotion of $\beta$-catenin/Foxo via inhibition of $\beta$-catenin/TCF slowed the rhTGF- $\beta 1$-induced wound closure but prevented epithelial cell differentiation into fibrotic mesenchymal phenotype. However, retardation of wound closure in Foxo KO cells suggested that induction of Foxo1 may enhance wound closure. In addition, a collagen detection assay identified the necessity of $\beta$-catenin binding to Foxo or TCF by altering $\beta$-catenin levels using F-TrCPE-cad in Foxo KO and TCF KO C1.1 cells. A role for TCF or Foxo in wound healing has already been reported. ${ }^{7,12,13}$ The current study demonstrated a novel mechanism for $\beta$ catenin/Foxo interaction in promoting wound repair when the $\beta$-catenin/TCF interaction is inhibited. Of more importance, inhibition of $\beta$-catenin/TCF leads to an epithelial repair, which we interpreted as being a result of shifting TGF- $\beta$ signaling from $\beta$-catenin/TCF to $\beta$-catenin/Foxo.

The current findings indicate shifting of $\beta$-catenin binding from TCF to Foxo through $\beta$-catenin/TCF inhibition in combination with TGF- $\beta$ in epithelial repair. However, deletion of Foxo1 provides indirect evidence for a dependence of repair on $\beta$-catenin/Foxo. Ideally, a $\beta$-catenin/Foxo inhibitor would be required to prove the role for $\beta$-catenin/ Foxo interaction. Surprisingly, we are unaware of any $\beta$-catenin/Foxo inhibitors developed so far. As a compromise, a Foxo activator, epigallocatechin 3-gallate, was used in the current study.

Kim 1 was used as a kidney tubular injury marker to assess the protection against kidney injury by promotion of $\beta$-catenin/Foxo. However, Kim 1 is also associated with kidney injury repair via recruiting and activating macrophages ${ }^{28}$ mostly contributing to a fibrotic repair associated with fibrosis when highly expressed.

Although TGF- $\beta$ is known to promote healing, the healing outcome is often one of tissue or organ fibrosis. Therefore, looking into TGF- $\beta$-mediated healing pathways has the potential to identify a therapeutic target to prevent fibrosis associated with healing and to increase the possibility of resumption of normal structure and function of the injured tissue. Cheon et $\mathrm{al}^{7,33}$ showed that growth factors regulated $\beta$-catenin-mediated TCFdependent transcriptional activation and increased the expression of $\alpha$-SMA, collagen type III, and fibronectin in hyperplastic cutaneous wounds. More importantly, by using $\beta$-catenin/TCF inhibitors along with TGF- $\beta$, epithelial repair was shown in tubular epithelial cell differentiation and in the UIR model. Thus, one of the major findings form the current study is the diversion of TGF$\beta$-mediated mesenchymal repair to epithelial repair.

Previous studies have suggested that TGF- $\beta$ promotes wound closure by increasing $\alpha$-SMA-mediated myofibroblast contraction, which can be mimicked by an in vitro collagen gel contraction assay. ${ }^{34,35}$ Fibrotic repair of the wound by TGF- $\beta$-induced overexpression of $\alpha$-SMA in myofibroblasts is the cause of excessive contraction and consequent destruction of normal mesenchymal structure. ${ }^{36}$ The in vitro study demonstrated that TGF- $\beta$ and ICG-001 (or iCRT3) efficiently inhibited the fibroblast-mediated excessive contractile activity of TGF- $\beta$. The shifting TGF$\beta$ signaling from $\beta$-catenin/TCF to $\beta$-catenin/Foxo resulted in an epithelial closure of the wound with less contraction, but this was sufficient to close the wound.

In summary, the current study shows that ICG-001 or iCRT3 efficiently inhibited TGF- $\beta$-induced mesenchymal repair and fibroblast contraction. This inhibition was associated with shifting of $\beta$-catenin binding from TCF to Foxo, whereby $\beta$-catenin/Foxo would promote epithelial repair. The interaction of $\beta$-catenin with TCF or Foxo may serve as a novel target for the treatment of kidney fibrosis.

\section{Acknowledgments}

We thank Virginia James for technical support and Kavitha Gowrishankar for reviewing the manuscript.

\section{Supplemental Data}

Supplemental material for this article can be found at http://doi.org/10.1016/j.ajpath.2021.03.005.

\section{References}

1. Bochaton-Piallat M-L, Gabbiani G, Hinz B: The myofibroblast in wound healing and fibrosis: answered and unanswered questions. F1000Research 2016, 5:F1000

2. Darby IA, Hewitson TD: Fibroblast differentiation in wound healing and fibrosis. Int Rev Cytol 2007, 257:143-179

3. Lichtman MK, Otero-Vinas M, Falanga V: Transforming growth factor beta (TGF-beta) isoforms in wound healing and fibrosis. Wound Repair Regen 2016, 24:215-222

4. Gewin LS: Renal tubule repair: is Wnt/beta-catenin a friend or foe? Genes 2018, 9:58 
5. Pressly JD, Hama T, Brien SO, Regner KR, Park F: TRIP13-deficient tubular epithelial cells are susceptible to apoptosis following acute kidney injury. Sci Rep 2017, 7:43196

6. Shinde AV, Humeres C, Frangogiannis NG: The role of alpha-smooth muscle actin in fibroblast-mediated matrix contraction and remodeling. Biochim Biophys Acta 2017, 1863:298-309

7. Cheon S, Poon R, Yu C, Khoury M, Shenker R, Fish J, Alman BA: Prolonged beta-catenin stabilization and tcf-dependent transcriptional activation in hyperplastic cutaneous wounds. Lab Invest 2005, 85: 416-425

8. Beyer C, Schramm A, Akhmetshina A, Dees C, Kireva T, Gelse K, Sonnylal S, de Crombrugghe B, Taketo MM, Distler O, Schett G, Distler JH: beta-Catenin is a central mediator of pro-fibrotic Wnt signaling in systemic sclerosis. Ann Rheum Dis 2012, 71:761-767

9. Cadigan KM, Waterman ML: TCF/LEFs and Wnt signaling in the nucleus. Cold Spring Harb Perspect Biol 2012, 4:a007906

10. Cheon SS, Cheah AYL, Turley S, Nadesan P, Poon R, Clevers H, Alman BA: beta-Catenin stabilization dysregulates mesenchymal cell proliferation, motility, and invasiveness and causes aggressive fibromatosis and hyperplastic cutaneous wounds. Proc Natl Acad Sci U S A 2002, 99:6973-6978

11. Novak A, Dedhar S: Signaling through beta-catenin and Lef/Tcf. Cell Mol Life Sci 1999, 56:523-537

12. Ponugoti B, Xu F, Zhang C, Tian C, Pacios S, Graves DT: FOXO1 promotes wound healing through the up-regulation of TGF-beta1 and prevention of oxidative stress. J Cell Biol 2013, 203:327-343

13. Hameedaldeen A, Liu J, Batres A, Graves GS, Graves DT: FOXO1, TGFbeta regulation and wound healing. Int J Mol Sci 2014, 15:16257-16269

14. Wuthrich RP, Glimcher LH, Yui MA, Jevnikar AM, Dumas SE, Kelley VE: MHC class II, antigen presentation and tumor necrosis factor in renal tubular epithelial cells. Kidney Int 1990, 37:783-792

15. Rao P, Pang M, Qiao X, Yu H, Wang H, Yang Y, Ren X, Hu M, Chen T, Cao Q, Wang Y, Khushi M, Zhang G, Wang YM, Heok P'ng C, Nankivell B, Lee VW, Alexander SI, Zheng G, Harris DC: Promotion of beta-catenin/Foxo1 signaling ameliorates renal interstitial fibrosis. Lab Invest 2019, 99:1689-1701

16. Liang CC, Park AY, Guan JL: In vitro scratch assay: a convenient and inexpensive method for analysis of cell migration in vitro. Nat Protocols 2007, 2:329-333

17. Yue PY, Leung EP, Mak NK, Wong RN: A simplified method for quantifying cell migration/wound healing in 96-well plates. J Biomol Screen 2010, 15:427-433

18. Skrypnyk NI, Harris RC, de Caestecker MP: Ischemia-reperfusion model of acute kidney injury and post injury fibrosis in mice. J Visualized Exp 2013, 78:50495

19. Zager RA, Johnson AC, Becker K: Acute unilateral ischemic renal injury induces progressive renal inflammation, lipid accumulation, histone modification, and "end-stage" kidney disease. Am J Physiol Renal Physiol 2011, 301:F1334-F1345

20. Hao S, He W, Li Y, Ding H, Hou Y, Nie J, Hou FF, Kahn M, Liu Y: Targeted inhibition of beta-catenin/CBP signaling ameliorates renal interstitial fibrosis. J Am Soc Nephrol 2011, 22:1642-1653

21. Qiao X, Rao P, Zhang Y, Liu L, Pang M, Wang H, Hu M, Tian X, Zhang J, Zhao Y, Wang XM, Wang C, Yu H, Guo F, Cao Q, Wang Y, Wang YM, Zhang GY, Lee VW, Alexander SI, Zheng G, Harris DCH: Redirecting TGF-beta signaling through the betacatenin/Foxo complex prevents kidney fibrosis. J Am Soc Nephrol 2018, 29:557-570
22. Sharma A, Yang WL, Ochani M, Wang P: Mitigation of sepsisinduced inflammatory responses and organ injury through targeting Wnt/beta-catenin signaling. Sci Rep 2017, 7:9235

23. Xiao H, Zeng Y, Wang Q, Wei S, Zhu X: A novel positive feedback loop between NTSR1 and Wnt/beta-catenin contributes to tumor growth of glioblastoma. Cell Physiol Biochem 2017, 43: $2133-2142$

24. Bartholome A, Kampkotter A, Tanner S, Sies H, Klotz LO: Epigallocatechin gallate-induced modulation of FoxO signaling in mammalian cells and C. elegans: FoxO stimulation is masked via PI3K/Akt activation by hydrogen peroxide formed in cell culture. Arch Biochem Biophys 2010, 501:58-64

25. Tian X, Zhang J, Tan TK, Lyons JG, Zhao H, Niu B, Lee SR, Tsatralis T, Zhao Y, Wang Y, Cao Q, Wang C, Wang Y, Lee VW, Kahn M, Zheng G, Harris DC: Association of betacatenin with P-Smad3 but not LEF-1 dissociates in vitro profibrotic from anti-inflammatory effects of TGF-beta1. J Cell Sci 2013, 126:67-76

26. Hoogeboom D, Essers MA, Polderman PE, Voets E, Smits LM, Burgering BM: Interaction of FOXO with beta-catenin inhibits beta-catenin/T cell factor activity. J Biol Chem 2008, 283: 9224-9230

27. Chang-Panesso M, Kadyrov FF, Lalli M, Wu H, Ikeda S, Kefaloyianni E, Abdelmageed MM, Herrlich A, Kobayashi A, Humphreys BD: FOXM1 drives proximal tubule proliferation during repair from acute ischemic kidney injury. J Clin Invest 2019, 129: $5501-5517$

28. Lim AI, Tang SC, Lai KN, Leung JC: Kidney injury molecule-1: more than just an injury marker of tubular epithelial cells? J Cell Physiol 2013, 228:917-924

29. Reinke JM, Sorg H: Wound repair and regeneration. Eur Surg Res 2012, 49:35-43

30. Sato M: Upregulation of the Wnt/beta-catenin pathway induced by transforming growth factor-beta in hypertrophic scars and keloids. Acta Derm Venereol 2006, 86:300-307

31. Peng J, Ramesh G, Sun L, Dong Z: Impaired wound healing in hypoxic renal tubular cells: roles of hypoxia-inducible factor-1 and glycogen synthase kinase 3 beta/beta-catenin signaling. J Pharmacol Exp Ther 2012, 340:176-184

32. Essers MA, de Vries-Smits LM, Barker N, Polderman PE, Burgering BM, Korswagen HC: Functional interaction between betacatenin and FOXO in oxidative stress signaling. Science 2005, 308: $1181-1184$

33. Cheon SS, Nadesan P, Poon R, Alman BA: Growth factors regulate beta-catenin-mediated TCF-dependent transcriptional activation in fibroblasts during the proliferative phase of wound healing. Exp Cell Res 2004, 293:267-274

34. Hasegawa T, Nakao A, Sumiyoshi K, Tsuchihashi H, Ogawa H: SB-431542 inhibits TGF-beta-induced contraction of collagen gel by normal and keloid fibroblasts. J Dermatol Sci 2005, 39: $33-38$

35. Kobayashi T, Liu X, Kim HJ, Kohyama T, Wen F-Q, Abe S, Fang Q, Zhu YK, Spurzem JR, Bitterman P, Rennard SI: TGF-betal and serum both stimulate contraction but differentially affect apoptosis in 3D collagen gels. Respir Res 2005, 6:141

36. Hinz B, Celetta G, Tomasek JJ, Gabbiani G, Chaponnier C: Alphasmooth muscle actin expression upregulates fibroblast contractile activity. Mol Biol Cell 2001, 12:2730-2741 